\title{
Cucurbitacins Elicit Anti-Platelet Activity via Perturbation of the Cytoskeleton and Integrin Function
}

Neline Kriek ${ }^{1}$ Sophie H. Nock ${ }^{2} \quad$ Tanya Sage $^{1} \quad$ Badrija Khalifa $^{2} \quad$ Alexander P. Bye $^{1} \quad$ Joanne L. Mitchell ${ }^{1}$ Steven Thomson ${ }^{2}$ Mark G. McLaughlin ${ }^{3}$ Sarah Jones $^{2}$ Jonathan M. Gibbins ${ }^{1}$ Amanda J. Unsworth ${ }^{1,20}$

${ }^{1}$ Institute for Cardiovascular and Metabolic Research, School of Biological Sciences, University of Reading, Reading, United Kingdom 2 Department of Life Sciences, Faculty of Science and Engineering, Manchester Metropolitan University, Manchester, United Kingdom

3 Department of Chemistry, Lancaster University, Lancaster, United Kingdom

Thromb Haemost 2022;122:1115-1129.

\author{
Address for correspondence Amanda J. Unsworth, PhD, Department \\ of Life Sciences, Faculty of Science and Engineering, John Dalton \\ Building, Manchester Metropolitan University, Manchester, M1 5GD, \\ United Kingdom (e-mail: a.unsworth@mmu.ac.uk).
}

\begin{abstract}
Cucurbitacins are dietary compounds that have been shown to elicit a range of antitumour, anti-inflammatory and anti-atherosclerotic activities. Originally identified as signal transducer and activator of transcription, STAT, inhibitors, a variety of mechanisms of action have since been described, including dysregulation of the actin cytoskeleton and disruption of integrin function. Integrin outside-in signalling and cytoskeletal rearrangements are critical for the propagation of stable thrombus formation and clot retraction following platelet adhesion at the site of vessel damage. The effects of cucurbitacins on platelet function and thrombus formation are unknown. We report for the first time anti-platelet and anti-thrombotic effects of cucurbitacins $B$, $E$ and $I$ in human platelets. Treatment of platelets with cucurbitacins resulted in attenuation of platelet aggregation, secretion and fibrinogen binding following stimulation by platelet agonists. Cucurbitacins were also found to potently inhibit other integrin- and cytoskeleton-mediated events, including adhesion, spreading and clot retraction. Further investigation of cytoskeletal dynamics found treatment with cucurbitacins altered cofilin phosphorylation, enhanced activation and increased $\mathrm{F}$ actin polymerisation and microtubule assembly. Disruption to cytoskeletal dynamics has been previously shown to impair integrin activation, platelet spreading and clot retraction. Anti-platelet properties of cucurbitacins were found to extend to a disruption of stable thrombus formation, with an increase in thrombi instability and deaggregation under flow. Our research identifies novel, anti-platelet and anti-thrombotic actions of cucurbitacins that appear to be linked to dysregulation of cytoskeletal dynamics and integrin function.
\end{abstract}

received

May 18, 2021

accepted after revision

November 6, 2021
DOI https://doi.org/ 10.1055/a-1788-5322. ISSN 0340-6245. (c) 2022. The Author(s).

This is an open access article published by Thieme under the terms of the Creative Commons Attribution-NonDerivative-NonCommercial-License, permitting copying and reproduction so long as the original work is given appropriate credit. Contents may not be used for commercial purposes, or adapted, remixed, transformed or built upon. (https://creativecommons.org/ licenses/by-nc-nd/4.0/)

Georg Thieme Verlag KG, Rüdigerstraße 14, 70469 Stuttgart, Germany 


\section{Introduction}

Natural compound libraries produced from plant products have identified a variety of promising compounds with a range of pharmacological properties for further development as therapeutic agents. ${ }^{1}$ The dietary flavonoids quercetin, tangeritin and nobiletin, ${ }^{2-4}$ derived from citrus fruits and dietary polyphenols, ${ }^{5}$ have all been shown to elicit antiplatelet properties via a variety of mechanisms ${ }^{3,4}$ including the regulation of cyclic guanosine monophosphate and cyclic adenosine monophosphate signalling pathways, reactive oxygen species generation and kinase-linked signalling pathways. $^{6}$

Cucurbitacins are a group of tetracyclic triterpernoid compounds that are found in members of the Cucurbitaceae plant family which includes pumpkins, squash, cucumber and melons. ${ }^{7}$

There are over 40 members of the cucurbitacin family, the most widely studied being cucurbitacins B, E and I, which have been shown to elicit a range of pharmacological properties including anti-cancer, anti-diabetic, antiinflammatory, anti-oxidant and anti-atherosclerotic activities. ${ }^{1,7,8}$ A variety of targets and mechanisms of action have been described including regulation of Janus kinase (JAK)/signal transducer and activator of transcription (STAT), ${ }^{9,10}$ cofilin, ${ }^{11,12}$ cyclins, ${ }^{13}$ cyclooxygenase $2^{14}$ and nuclear factor-kappa B activity and function. ${ }^{15}$ Cucurbitacins have also been identified to cause dysregulation of actin cytoskeleton dynamics ${ }^{11,12,16}$ and disruption of integrin function. ${ }^{17,18}$

Platelets perform a critical protective role in haemostasis preventing blood loss upon injury. Platelet activation and thrombosis, however, can also be triggered by arterial damage following rupture or erosion of an atherosclerotic plaque resulting in myocardial infarction or stroke. Drugs which disrupt platelet function therefore represent the cornerstone of antithrombotic treatment in cardiovascular disease patients.

Integrin outside-in signalling and cytoskeletal rearrangements are critical events in platelet activation and are required for the formation of stable thrombi. ${ }^{19-21}$ The initial activation of platelets is followed by rearrangement of the platelet cytoskeleton to form filopodia and lamellipodia that leads to platelet shape change and spreading. ${ }^{22}$ Integrin adhesions support platelet aggregation via fibrinogen binding to integrin $\alpha \operatorname{IIb} \beta 3$, and clot retraction is then driven through integrin outside-in signalling and cytoskeleton contraction that subsequently stabilises and supports the growing thrombus. ${ }^{20,23}$

We have identified anti-platelet and anti-thrombotic activity of cucurbitacins B, E and I. We show that these inhibitory effects are associated with dysregulation of platelet cytoskeletal dynamics and the subsequent inhibition of platelet integrin $\alpha$ IIb $\beta 3$-mediated aggregation and thrombus formation thus identifying the future potential for the development of cucurbitacin-derived compounds as novel anti-platelet and anti-thrombotic agents.

\section{Methods}

\section{Reagents}

Cucurbitacins B, E and I were purchased from Cambridge Bioscience (Cambridge, United Kingdom). Adenosine diphosphate (ADP), thrombin receptor-activating peptide-6 (TRAP6 ), fibrinogen, fibronectin and laminin were purchased from Sigma Aldrich (Poole, United Kingdom). Horm collagen was purchased from Nycomed, Austria, and Labmedics, crosslinked collagen-related peptide (CRP-XL) was purchased from Prof. R. Farndale (University of Cambridge, United Kingdom).

Primary phospho-antibodies for (P) cofilin Ser3 (\#3311), (P) LIMK T508/505 (\#3841), (P) myosin light chain (MLC) S19 (\#3671) and (P) vasodilator-stimulated phosphoprotein (VASP) S157 (\#3111), were purchased from New England Biosciences (Cell Signaling Hitchin, United Kingdom). $\beta$-actin (C4) antibody was purchased from Santa Cruz (\# sc47778), tubulin antibodies (\#ATN02) from Cytoskeleton Inc. (Denver, United States) and $\beta$-tubulin (\# PAS-16863) from Invitrogen and platelet factor 4 (PF4) (\#ab129183) antibody from Abcam (Cambridge, United Kingdom). Fluorophoreconjugated secondary antibodies, FURA-2AM calcium indicator dye and Alexa-488 conjugated phalloidin were purchased Life Technologies (Paisely, United Kingdom).

\section{Platelet Preparation}

For human experiments, blood was obtained from consenting aspirin-free healthy volunteers following procedures approved by the Manchester Metropolitan University and University of Reading Research Ethics Committees. Blood was collected into $4 \%(\mathrm{w} / \mathrm{v}$ ) sodium citrate and then mixed with acid citrate dextrose (ACD) (29.9 mM trisodium citrate, $113.8 \mathrm{mM}$ glucose and $2.9 \mathrm{mM}$ citric acid [pH 6.4]) if washed platelets were prepared. Platelet-rich plasma (PRP) was prepared by centrifugation at $100 \times \mathrm{g}$ for 20 minutes at room temperature. ADP sensitive washed platelets were prepared from PRP (containing 1:8 v/v ACD) by centrifugation at $350 \times g$ for 20 minutes followed by resuspension in modified Tyrode's-HEPES buffer (134 mM NaCl, $0.34 \mathrm{mM}$ $\mathrm{Na}_{2} \mathrm{HPO}_{4}, 2.9 \mathrm{mM} \mathrm{KCl}, 12 \mathrm{mM} \mathrm{NaHCO} 3,20 \mathrm{mM} \mathrm{N}$-2-hydroxyethylpiperazine-N-2-ethanesulfonic acid, $5 \mathrm{mM}$ glucose and $1 \mathrm{mM} \mathrm{MgCl}_{2}, \mathrm{pH} 7.3$ ) and used immediately.

\section{Platelet Aggregation}

Aggregation of human washed platelets was measured by optical aggregometry, using a Helena AggRAM aggregometer (Helena Biosciences, Gateshead, United Kingdom) as described previously, ${ }^{24}$ following pre-treatment of platelets with cucurbitacins or vehicle ( $0.1 \%$ dimethyl sulfoxide [DMSO]) for 10 minutes prior to agonist stimulation. Agonists used to stimulate platelet aggregation include $1 \mu \mathrm{g} / \mathrm{mL}$ collagen, $1 \mu \mathrm{g} / \mathrm{mL}$ CRP-XL, $1 \mu \mathrm{M}$ TRAP-6 and $10 \mu \mathrm{M}$ ADP.

\section{Granule Secretion and Fibrinogen Binding by Flow Cytometry}

Flow cytometry was used to examine $\alpha$ and dense-granule secretion and affinity up-regulation of the integrin $\alpha \operatorname{IIb} \beta 3$ by 
detecting levels of P-selectin exposure, CD63 exposure and fibrinogen binding, respectively. Following incubation with the cucurbitacins or vehicle for 10 minutes and stimulation with different platelet agonists (ADP, CRP-XL and TRAP-6), platelets were incubated at room temperature for 20 minutes with either a phycoerythrin (PE)/Cy5 anti-human CD62P (Pselectin), PE anti-human CD63 or fluorescein isothiocyanate (FITC)-labelled anti-fibrinogen antibody. Reactions were stopped after 20 minutes, and platelets fixed by addition of $0.2 \%(\mathrm{v} / \mathrm{v})$ paraformaldehyde. Data for 5,000 events were collected using either a BD Accuri C6 flow cytometer and analysed using the CFlow Sampler software or MACsQuant Analyser 16 flow cytometer and analysed using FlowLogic software as described previously. ${ }^{25}$

\section{PF4 Release}

Washed platelets were pre-incubated for 10 minutes with 10 $\mu \mathrm{M}$ cucurbitacins B, E or I or vehicle and stimulated with a concentration of TRAP- 6 that elicited full aggregation for the vehicle but showed reduced aggregation with the cucurbitacins. After 3 minutes aggregation, the reaction was stopped by addition of prostaglandin I2 $(500 \mathrm{ng} / \mathrm{mL})$, platelets were pelleted and PF4 in the supernatants detected by Western blot analysis.

\section{Microvesicle Formation and Annexin V Binding}

Flow cytometry was used to investigate platelet microvesicle formation and phosphatidylserine (PS) exposure. Washed platelets were resuspended in $1 \times$ annexin $\mathrm{V}$ binding buffer (BD Biosciences) and treated with the cucurbitacins or vehicle for 10 minutes prior to stimulation with $10 \mu \mathrm{M}$ A23817 for 10 minutes at room temperature. PE-conjugated anti-human CD41a antibody was used to identify platelets and plateletderived microvesicles. FITC-conjugated annexin V (Ann) was used to detect surface PS exposure on platelet-derived microvesicles and platelets. Microvesicles were gated from platelets based on forward scatter (FSC) profile as described previously. ${ }^{26}$ Platelet annexin $\mathrm{V}$ binding was determined from data collected for 10,000 events in the platelet gate, and number of platelet-derived microvesicles (low FSC, CD41 + /Ann + events) was determined per $1 \mu \mathrm{L}$, using a MACsQuant Analyser 16 flow cytometer and analysed using FlowLogic software.

\section{Mobilisation of Intracellular Calcium}

PRP was loaded with Fura-2 AM $(2 \mu \mathrm{M})$ for 1 hour at $30^{\circ} \mathrm{C}$ and then washed by centrifugation at $350 \times \mathrm{g}$ for 20 minutes and resuspended in Tyrode's-HEPES buffer containing $0.4 \mathrm{U} / \mathrm{mL}$ apyrase. Fura-2 loaded platelets were incubated with inhibitors or vehicle at $37^{\circ} \mathrm{C}$ prior to addition of agonists. Fluorescence measurements with excitation at 340 and $380 \mathrm{~nm}$ and emission at $510 \mathrm{~nm}$ were recorded over a period of 5 minutes using a FlexStation (Molecular Devices, Winnersh, United Kingdom). $\left[\mathrm{Ca}^{2+}\right]_{i}$ was estimated using the ratio of the 340 and $380 \mathrm{~nm}$ excited signals and the method of Grynkiewicz et al was utilised. ${ }^{27-29}$

\section{Platelet Adhesion and Spreading}

Washed platelets at $2 \times 10^{7} \mathrm{cell} / \mathrm{mL}$ were exposed to collagen $(100 \mu \mathrm{g} / \mathrm{mL})$, fibrinogen $(100 \mu \mathrm{g} / \mathrm{mL})$, fibronectin $(200 \mu \mathrm{g} / \mathrm{mL})$ or laminin $(50 \mu \mathrm{g} / \mathrm{mL})$-coated 96 -well assay plates and allowed to adhere for 45 minutes at $37^{\circ} \mathrm{C}$ following treatment with or without cucurbitacins. Non-adherent platelets were removed by washing in phosphate-buffered saline (PBS) before fixing with $10 \%$ formyl saline for 10 minutes. The effect on cucurbitacin treatment on platelet spreading was also determined post-adhesion, with spreading assays performed on platelets allowed to adhere to fibrinogen-coated surface for 20 minutes prior to addition of cucurbitacins $B, E$ or I. Non-adherent platelets were removed, and adhered platelets treated with cucurbitacins B, E or I and left to continue to spread for an additional 25 minutes before fixing as described above. The wells were then washed and labelled with DiOC6, to visualise the membrane, or FITC-conjugated phalloidin, to visualise the actin cytoskeleton. Fluorescence images of adherent platelets were captured with the $20 \times$ objective lens of an ImageXpress Pico high content imaging system and manually counted using CellReporterXpress software (Molecular Devices, WInnersh, UK) or imaged on a Celena $\mathrm{S}$ logos digital imaging system (Labtech International, Sussex, UK) and manually counted using ImageJ software. All platelets per field of view were counted, with a minimum of 100 platelets per field of view required for the dataset to be included.

\section{Thrombus Formation under Flow In Vitro}

Thrombus formation on and platelet interaction with immobilised type I collagen was performed as described previously ${ }^{25}$ using the VenaFlux Platform and Vena8Fluor+ Biochips (Cellix). ${ }^{30}$ Channels were coated overnight at $4{ }^{\circ} \mathrm{C}$ with collagen, then blocked with $1 \%$ bovine serum albumin (BSA)/PBS for 1 hour and replaced with PBS. Human blood was collected into sodium citrate $(4 \% \mathrm{w} / \mathrm{v})$. Platelets in whole blood were incubated with $10 \mathrm{mM}$ DiOC6 (Thermo Fischer Scientific) for 10 minutes. Perfusion was performed for 10 minutes (human) at $37^{\circ} \mathrm{C}$ with an arterial shear rate of 45 dynes $/ \mathrm{cm}^{2}$ / 1,000 second. Human thrombus formation was visualised using a $20 \times$ magnification lens on a Nikon A1-R confocal microscope with images taken over a 10-minute time period. Images were analysed using Fiji Image software to determine surface area coverage and fluorescence intensity over time as described previously, ${ }^{31-34}$ and the thrombus instability index $(\Delta \mathrm{Sd} / \Delta \mathrm{T}(\%))$ was calculated. $\Delta \mathrm{Sd} / \Delta \mathrm{T}(\%)$ is the change in surface distribution relative to change in time and is described in detail by Pugh et al. ${ }^{31,32} \mathrm{~A}$ low $\Delta \mathrm{Sd} / \Delta \mathrm{T}(\%)$ value represents stable thrombi where there is little change in fluorescent intensity over time. In contrast, non-stable thrombi or rolling platelets display higher $\Delta \mathrm{Sd} / \Delta \mathrm{T}(\%)$ values, as platelets detach.

\section{Flow Cytometry Actin Polymerisation Assay}

Human washed platelets were resuspended at $4 \times 10^{8} / \mathrm{mL}$ and treated with cucurbitacins B, E or I $(0.1,1,10 \mu \mathrm{M})$ or vehicle ( $0.1 \%$ DMSO) for 10 minutes prior to stimulation with or without $10 \mu \mathrm{M}$ TRAP- 6 for 3 minutes at room temperature before fixing with an equal volume of $2 \%$ formyl saline. Samples were centrifuged at $1,000 \times g$ for 10 minutes at $4^{\circ} \mathrm{C}$. Supernatant was discarded and pellet resuspended in PBS, before a repeat centrifugation step. The pellet was 
resuspended in BD Phosflow Perm Buffer III and incubated on ice for 30 minutes. Platelets were then washed three times as described above using PBS and a centrifugation step of $1,000 \times \mathrm{g}$. The pellet was resuspended in PBS containing 1:500 dilution of Alexa488-conjugated phalloidin and incubated for 1 hour in the dark. Phalloidin staining (marker of polymerised actin) was then determined by flow cytometry using a BD Accuri C6 plus and data analysed using the CFlow Sampler software as described previously. ${ }^{25}$

\section{F/G Actin Ratio Actin Polymerisation Assays}

Human washed platelets were resuspended at $8 \times 10^{8} / \mathrm{mL}$ and treated with cucurbitacins B, E or I $(10 \mu \mathrm{M})$ or vehicle (0.1\% DMSO) for 10 minutes prior to stimulation with or without $10 \mu \mathrm{M}$ TRAP-6 for 3 minutes at room temperature. Following stimulation, an equal volume of $(2 \times)$ actin stabilisation buffer (0.2M PIPES, pH 6.9, 60\% glycerol, $10 \%$ DMSO, $2 \mathrm{mM}$ MgSO4, $2 \mathrm{mM}$ EGTA, $2 \%$ Triton X-100, $1 \mathrm{mM}$ PMSF, $2 \mathrm{mM} \mathrm{NaVO4}, 10 \mu \mathrm{g} / \mathrm{mL}$ leupeptin, $10 \mathrm{ug} / \mathrm{mL}$ aprotinin, $1 \mathrm{ug} / \mathrm{mL}$ pepstatin A, $2 \mathrm{mM}$ ATP) was added to the samples at $4^{\circ} \mathrm{C}$. Samples were centrifuged at $16,000 \times \mathrm{g}$ for 75 minutes at $4^{\circ} \mathrm{C}$ and the supernatant (containing monomeric $G$ actin) removed. The pellet (containing polymerised $\mathrm{F}$ actin) was then resuspended in an equivalent volume of actin depolymerisation buffer (0.1 M PIPES, pH 6.9, $1 \mathrm{mM}$ MgSO4, $10 \mathrm{mM} \mathrm{CaCl2}, 5 \mu \mathrm{M}$ cytochalasin D) containing cytochalasin $\mathrm{D}(5 \mu \mathrm{M})$. Both $\mathrm{G}$ actin and $\mathrm{F}$ actin samples were then lysed in Laemmli sample buffer and boiled. Samples were separated by sodium dodecyl sulfate polyacrylamide gel electrophoresis electrophoresis and transferred to polyvinylidene difluoride membranes by Western blotting.

\section{Cytoskeleton Imaging}

Washed platelets were resuspended to $4 \times 10^{7}$ cells $/ \mathrm{mL}$ and incubated with cucurbitacins or vehicle control for 10 minutes at room temperature. Reactions were stopped with an equal volume of $10 \%$ formalin and samples were pelleted onto slides as described in the work by Khan et al. ${ }^{35}$ Samples were permeabilised with $0.1 \%$ Triton for 5 minutes before washing $3 \times$ with PBS. Wells were blocked with $1 \%$ BSA/PBS for 1 hour at room temperature, before samples were stained with $\beta$ Tubulin Polyclonal Antibody (Invitrogen) with a dilution of 1:200 in BSA/PBS for 1 hour at room temperature. Samples were washed before staining with goat anti-rabbit AlexaFluor 647 antibody (Invitrogen) and AlexaFluor 488 phalloidin (1:750) for 1 hour at room temperature. Slides were washed, mounted and imaged on a Zeiss Imager M2 100× lens.

\section{Immunoblotting}

Immunoblotting was performed using standard techniques as described previously. ${ }^{32,33}$ Proteins were detected using fluorophore-conjugated secondary antibodies and visualised using a Typhoon Fluorimager and Image Quant software (GE Healthcare). Band intensities were quantified and levels of the total protein were used to normalise the phosphorylation data using Image Quant software and ImageJ.

\section{Statistics}

All experiments were performed using blood from a minimum of three separate donors. Statistical analyses of the data were performed using GraphPad Prism software. When comparing two sets of data, an unpaired, two-tailed Student's $t$-test (simple) statistical analysis was used. If more than two means were present, significance was determined by one-way or two-way analysis of variance followed by Bonferroni or Tukey correction (multiple). Where data were normalised, statistical analysis was performed prior to normalisation and also using the non-parametric Wilcoxon signed-rank test. A $p$-value of $\leq 0.05$ was considered statistically significant. Unless stated otherwise, values were expressed as mean \pm standard error of the mean.

\section{Results}

\section{Cucurbitacins Attenuate Platelet Aggregation and Granule Secretion}

Platelet aggregation mediated through activation of integrin $\alpha$ IIb $\beta 3$ and the formation of inter-platelet fibrinogen bridges is a key step in the process of thrombus formation. It has been previously described that treatment with cucurbitacins disrupts integrin function in cancer cells through inactivation of the integrin $\alpha$ subunit. ${ }^{17,18}$ Light transmission aggregometry was used to determine the effect of treatment of human washed platelets with increasing concentrations of cucurbitacins on platelet aggregation. We found that treatment with cucurbitacin B, E or I caused a dose-dependent attenuation of platelet aggregation to a range of platelet agonists, including collagen, CRP-XL, ADP and protease-activated receptor-1 activating peptide TRAP-6 (-Fig. 1). With approximately 30 to $50 \%$ inhibition of aggregation observed following treatment with the highest concentration tested of each cucurbitacin $(10 \mu \mathrm{M})$. Consistent with an inhibition of platelet aggregation, integrin $\alpha \operatorname{Ilb} \beta 3$ activation, assessed by fibrinogen binding, was reduced following treatment of washed platelets with cucurbitacin B, E or I $(10 \mu \mathrm{M})$ prior to stimulation with TRAP-6 or ADP. Interestingly, no inhibition of fibrinogen binding was observed following stimulation with $1 \mu \mathrm{M}$ CRP-XL (-Fig. 2) despite the observed inhibition of aggregation responses at the same concentration. This may be due to assay differences and/or the sensitivity of the CRP-XL used.

In contrast to integrin $\alpha$ IIb $\beta 3$ activation, P-selectin exposure a marker of $\alpha$ granule secretion, another key process in platelet activation and thrombus formation, was found to be unaffected by treatment with cucurbitacin B, E or I (-Fig. 2). We therefore determined whether this was also the case for secretion of dense granules. Interestingly, in contrast to Pselectin exposure, we did see an inhibition of TRAP-6induced CD63 exposure (a component of dense granules) following treatment with cucurbitacins B, E and I $(10 \mu \mathrm{M})$ (-Fig. 2D) indicating dense granule secretion is affected. It has been previously described that P-selectin exposure is not always representative of $\alpha$ granule secretion. ${ }^{36}$ We therefore analysed secretion of soluble mediator and $\alpha$ granule component PF4 following stimulation by TRAP-6. In contrast to P- 

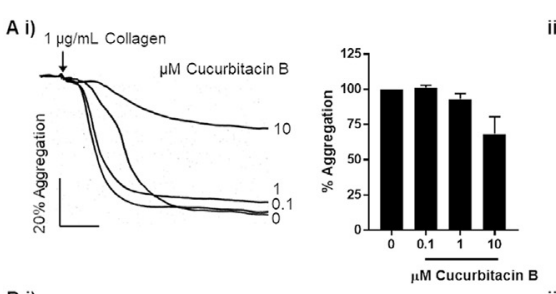

B i) $1 \mu \mathrm{g} / \mathrm{mL}$ CRP-XL
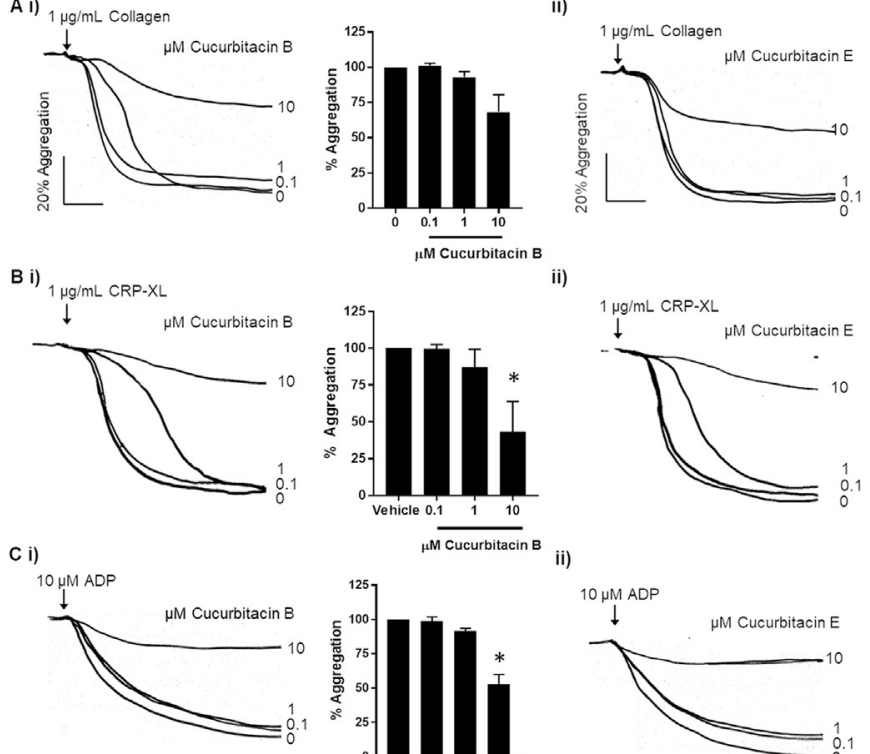

$\mu \bar{M}$ Cucurbitacin B

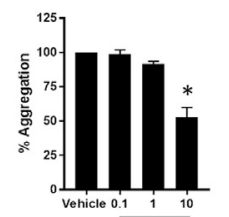

Di) 1 MM TRAPG
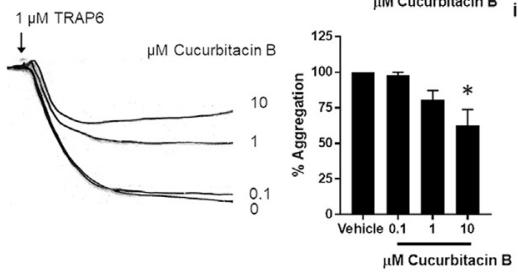

$10 \mu \mathrm{M}$ ADP
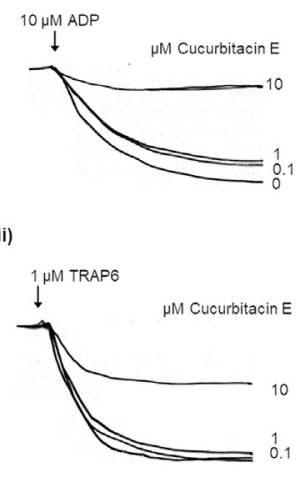
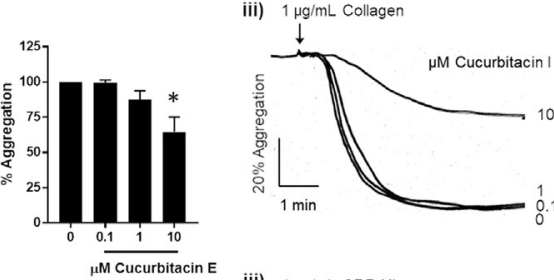

iii) $1 \mu \mathrm{g} / \mathrm{mL}$ CRP-XL
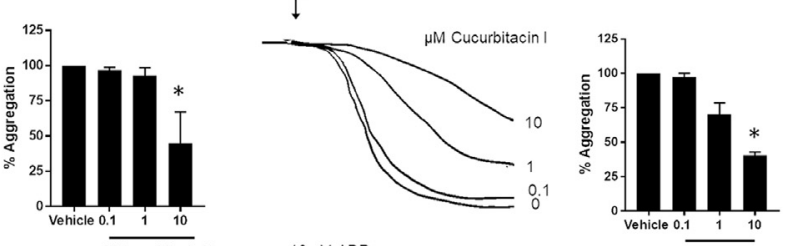

iii)


Fig. 1 Cucurbitacin B, E and I inhibit platelet aggregation. Washed human platelets were pre-treated with increasing concentrations of cucurbitacin (i) B, (ii)

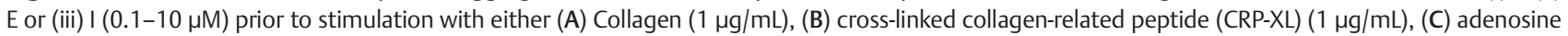
diphosphate (ADP) $(10 \mu \mathrm{M})$, (D) thrombin receptor-activating peptide-6 (TRAP-6) $(1 \mu \mathrm{M})$, and aggregation monitored using optical light transmission aggregometry, representative traces and quantified data shown. Results are mean + standard error of the mean (SEM) for $n \geq 3$, ${ }^{*}$ indicates $p<0.05$ in comparison to vehicle controls, where normalised data shown, statistics were performed prior to normalisation.

selectin exposure a reduction in PF4 release was observed following cucurbitacin treatment, under the same conditions where cucurbitacins cause inhibition of platelet aggregation. Indicating that both $\alpha$ and dense granule secretion appear to be affected following treatment with cucurbitacins.

We next determined whether cucurbitacins caused inhibition of platelet functional responses as a result of alteration of a global regulatory mechanism such as activation of central signalling components. Interestingly, treatment of platelets with cucurbitacin B, E or I at concentrations that attenuate platelet aggregation, integrin function and granule secretion did not alter cytosolic calcium mobilisation following stimulation with TRAP-6 $(1 \mu \mathrm{M})$ a key regulator of downstream platelet signalling pathways and platelet activation (-Supplementary Fig. S1, available in the online version). Indicating cucurbitacins inhibit platelet function via regulation of processes that occur downstream of calcium signalling.

\section{Cucurbitacin Treatment Inhibits Platelet Adhesion and Spreading}

Platelet adhesion and activation on extracellular matrix components is mediated by integrin activity. Following platelet adhesion, activation and subsequent fibrinogen binding, clustering of integrin $\alpha \operatorname{IIb} \beta 3$ occurs and outside-in signalling is initiated leading to platelet cytoskeleton rearrangements, platelet shape change and spreading. The effect of cucurbitacins on platelet adhesion and spreading was therefore determined on a range of platelet adhesive surfaces.

As shown in - Fig. 3 (and - Supplementary Fig. S2, available in the online version), treatment of platelets with increasing concentrations of the different cucurbitacins caused a decrease in the ability of the platelets to adhere (cell count) and spread (surface area) on fibrinogen, collagen, fibronectin and laminin-coated surfaces (inhibition of $\sim 20-$ 50\%). IC50 (inhibitory concentration) values $(<1 \mu \mathrm{M})$ showed cucurbitacins B, E and I to inhibit platelet adhesion and spreading on the different adhesive surfaces more potently than the concentrations required to achieve $50 \%$ inhibition of aggregation or integrin activation $(10 \mu \mathrm{M})$. Attenuation of platelet adhesion and spreading was not due to reduction in surface expression levels of integrin $\alpha \operatorname{IIb} \beta 3$ and other platelet surface receptors, as surface expression levels of $\alpha \mathrm{IIb}, \beta 3, \alpha 2, \beta 1$ and glycoprotein VI were unaltered following treatment with cucurbitacins (-Fig. 3C).

The observed impairment of platelet adhesion and spreading on multiple adhesive surfaces indicates global disruption of adhesive integrin receptor signalling or cytoskeletal rearrangements. In further support of this, analysis of platelet spreading on glass coverslips identified no significant alteration in platelet adhesion following treatment with 


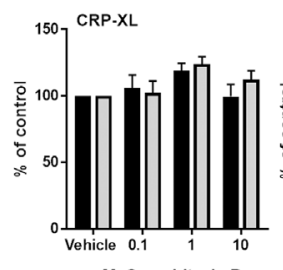

B i) $\mu \mathrm{M}$ Cucurbitacin B

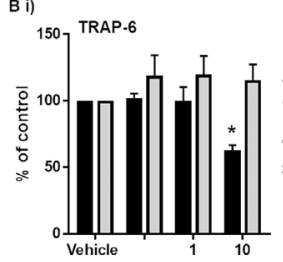
$\mu \mathrm{M}$ Cucurbitacin B

C i)

i) 150 ADP

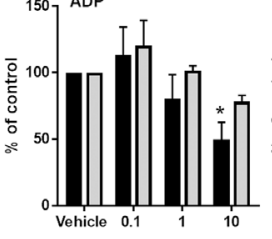

$\mu \mathrm{M}$ Cucurbitacin

D i)
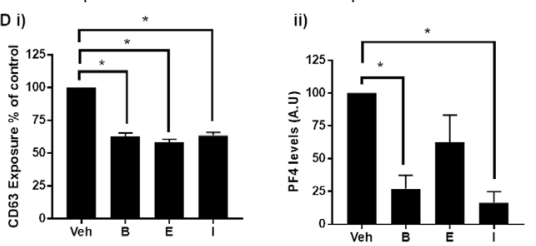

Fig. 2 Cucurbitacin B, E and I inhibit integrin $\alpha$ llb $\beta 3$ activation but not $\alpha$ granule secretion. Washed human platelets were pre-treated with increasing concentrations of cucurbitacin $B$, E or I $(0.1-10 \mu \mathrm{M})$ prior to stimulation with either $(A)$ cross-linked collagen-related peptide (CRP-XL) (1 $\mu \mathrm{g} / \mathrm{mL})$, (B) thrombin receptor-activating peptide-6 (TRAP-6) $(1 \mu \mathrm{M})$ or (C) adenosine diphosphate (ADP) $(10 \mu \mathrm{M})$ and $\alpha$ granule secretion determined by monitoring P-selectin exposure (grey) and integrin activation measured as fibrinogen binding (black) determined using flow cytometry, quantified data shown. (D) Washed human platelets were pre-treated with cucurbitacin B, E or I ( $10 \mu \mathrm{M})$ prior to stimulation with TRAP-6 $(1 \mu \mathrm{M})$ and (i) dense granule secretion determined by monitoring CD63 surface expression via flow cytometry and (ii) release of soluble $\alpha$ granule contents determined by measuring platelet factor 4 (PF4) levels of the platelet releasate by Western blotting, quantified data shown. Results are mean + standard error of the mean (SEM) for $n \geq 3$, ${ }^{*}$ indicates $p<0.05$ in comparison to vehicle controls, where normalised data shown, statistics were performed prior to normalisation.

either cucurbitacin B, E or I $(1 \mu \mathrm{M})$, but did cause a significant reduction in platelet surface area (cucurbitacin $B$ and $E$ ), indicating an inhibition of the platelets to undergo normal shape change and spreading (- Supplementary Fig. S3A, available in the online version). In addition, when platelets were left to adhere to a fibrinogen-coated surface for 20 minutes prior to treatment with cucurbitacins, no alteration in platelet adhesion was observed, indicating no alteration in the ability of platelets to maintain adhesion and outside-in signalling. However, some reduction in spreading (cell size - surface area) was observed following treatment with cucurbitacin $B$ and $\mathrm{E}(10 \mu \mathrm{M})$, following adhesion, indicating some disruption to cytoskeletal dynamics following treatment with the cucurbitacins (-Supplementary Fig. S3B, available in the online version).

\section{Cucurbitacins Inhibit Stable Thrombus Formation}

Having observed an inhibitory effect of cucurbitacins on platelet adhesion and aggregation, the effect of the cucurbitacins on thrombus formation was assessed in whole blood under arterial shear conditions in collagen-coated microfluidic chambers. Human whole blood was pre-incubated with vehicle control or cucurbitacin B, E or I $(10 \mu \mathrm{M})$ for 10 minutes and perfused over collagen-coated $(100 \mu \mathrm{g} / \mathrm{mL})$ Vena8 biochips at arterial shear rate ( $45 \mathrm{dyn} / \mathrm{cm}^{3}$ for 10 minutes). Interestingly, while initial adhesion to collagen appeared to be unaffected, cucurbitacin-treated platelets exhibited a reduced ability to form stable platelet aggregates and stable thrombi (-Fig. 4A). In the presence of vehicle, platelets accumulated on collagen fibres while continuously contracting into dense stable thrombi. In contrast, treatment with cucurbitacins prevented retraction of platelet aggregates, which consequently remained unstable, appeared loose and were prone to disaggregation with several micro-thrombi observed detaching from the site of adhesion. In support of this, surface area coverage was significantly increased in cucurbitacin B, E or I treated samples compared with vehicle-treated control ( $\mathbf{- F i g . ~ 4 B ) ~ i n d i c a t i n g ~}$ their inability to contract and form dense thrombi. Fluorescence intensity, used to analyse thrombus formation, was unaffected following treatment with cucurbitacins B, E or I ( - Fig. 4C). This anomaly could be attributed to the increased surface area coverage but lack of stable dense thrombi formation compared with vehicle controls. To quantify thrombus stability, the thrombus instability index $\Delta \mathrm{Sd} / \Delta \mathrm{T}(\%)^{31,34}$ was used ( - Fig. 4D). A significant increase in $\Delta \mathrm{Sd} / \Delta \mathrm{T}$ (\%) following treatment with cucurbitacin B, E or I was observed compared with vehicle-treated controls. These observations indicate that treatment with cucurbitacins prevents platelets from being able to form stable thrombi under flow conditions.

\section{Cucurbitacins Attenuate Clot Retraction}

The significant reduction in the ability of platelets to form stable thrombi following incubation with cucurbitacins indicates inhibited clot retraction. The effect of cucurbitacins on clot retraction was therefore explored (-Fig. 5). Pre-incubation of platelets with cucurbitacins B, E or I resulted in an increase in clot weight and therefore an inhibition of clot retraction after 90 minutes following stimulation with thrombin, compared with vehicle-treated controls ( $0.1 \% \mathrm{v} / \mathrm{v}$ DMSO). Lack of stable thrombus formation and inhibition of clot retraction is typical of attenuated outside-in signalling via integrin $\alpha$ IIb $\beta 3$ or disruption of cytoskeletal rearrangements preventing platelets from changing shape and 'contracting' within a thrombus.

\section{Cucurbitacins Alter Platelet Cytoskeleton Dynamics}

During platelet activation, integrin activity is tightly associated with cytoskeletal dynamics and the platelet cytoskeleton has been shown to regulate platelet dense and $\alpha$ granule secretion. $^{36-38}$ The anti-cancer cytotoxic properties of some of the cucurbitacins have been shown to be associated with disruption of cytoskeletal dynamics which ultimately leads to an inhibition of cell division and/or cell migration. ${ }^{11,12,16,39}$ To determine whether cucurbitacins altered cytoskeleton remodelling in platelets, we monitored 
A i)

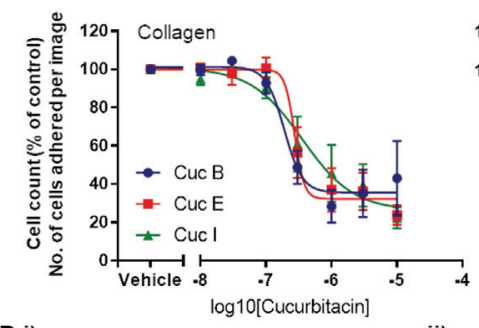

B i)

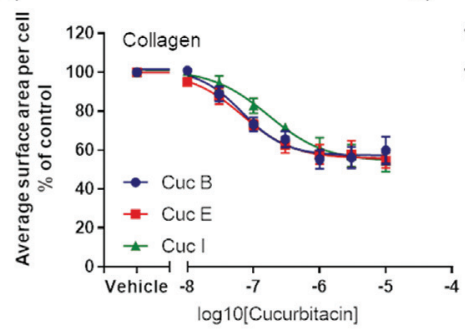

ii)
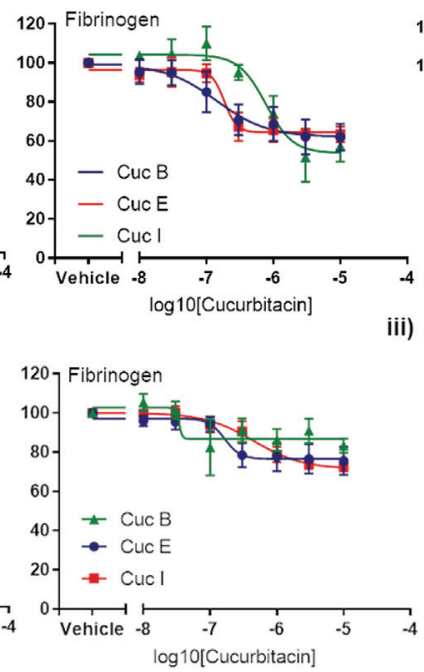

iii)

iii)

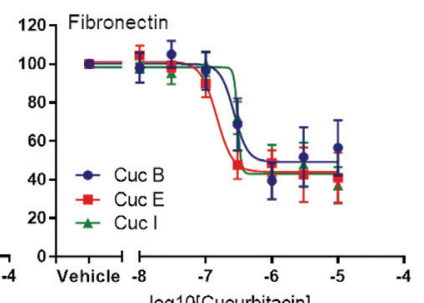

$\log 10[$ Cucurbitacin]

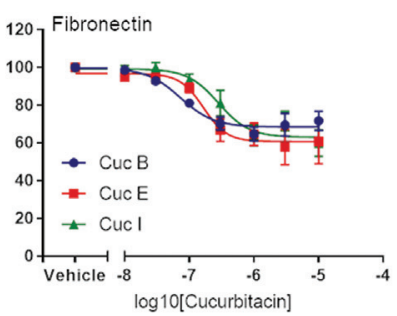

iv) iv)


C)

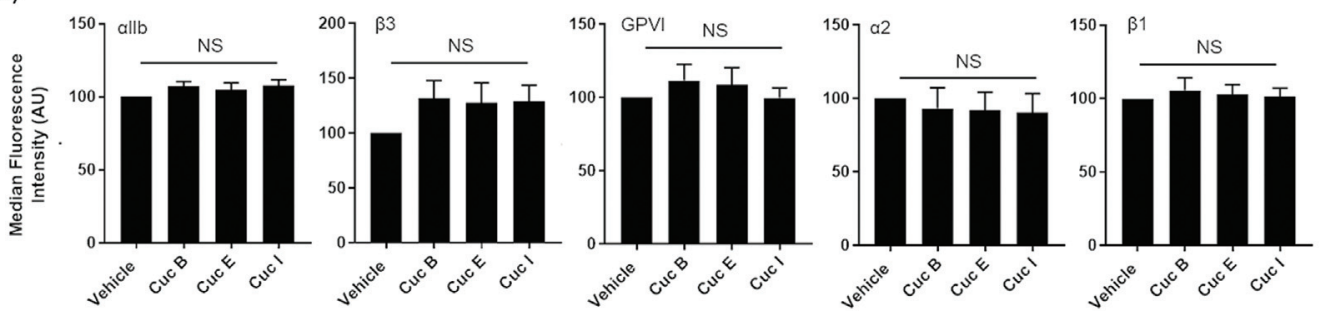

Fig. 3 Cucurbitacins inhibit platelet adhesion and spreading. Human washed platelets pre-treated for 10 minutes with or without increasing concentrations


(iv) laminin $(50 \mu \mathrm{g} / \mathrm{mL})$-coated coverslips and (A) adhesion and (B) spreading determined after 45 minutes. Platelets were labelled with DiOC6 and fluorescence images of adherent platelets were captured with the $20 \times$ objective lens of an ImageXpress Pico high content imaging system and manually counted using CellReporterXpress software. (A) Number of platelets adhered (adhesion) were counted per field of view for each condition and the number of cells adhered expressed as a percentage of the vehicle-treated control. (B) Extent of spreading determined by calculating the average surface area per platelet and expressed as a percentage of vehicle-treated control. All platelets per field of view counted, with a minimum of 100 platelets per field of view required for the dataset to be included. (C) Receptor surface expression levels of $\alpha$ llb, $\beta 3$, glycoprotein VI (GPVI), $\alpha 2$ and $\beta 1$ were determined by flow cytometry using fluorophore-conjugated antibodies and by measuring median fluorescence intensity following treatment with or without $10 \mu \mathrm{M}$ cucurbitacin $\mathrm{B}$, E or I. Data expressed as percentage of vehicle control. Results are mean + standard error of the mean (SEM) for $n \geq 3,{ }^{*}$ indicates $p<0.05$., NS indicates $p>0.05$ in comparison to vehicle controls, where normalised data shown, statistics were performed prior to normalisation.

actin polymerisation in platelets stimulated with or without $10 \mu \mathrm{M}$ TRAP-6 following treatment with and without cucurbitacin B, E and I. We identified that treatment with cucurbitacins resulted in an increase in the platelet pool of polymerised actin (F actin) (- Fig. 6A) and an increase in the $\mathrm{F} / \mathrm{G}$ actin ratio in both resting and stimulated platelets (TRAP6) compared with vehicle-treated controls (-Fig. $\mathbf{6 B}$ and C) indicating that cucurbitacins alter actin turnover. A decrease in tubulin:microtubule ratio was also observed in both resting and stimulated platelets (TRAP-6) following cucurbitacin treatment (-Fig. 6D and E). Observation of cucurbitacin mediated changes in the absence of TRAP6, suggests that dysregulation of platelet cytoskeleton dynamics following treatment with cucurbitacins occurs independently of platelet activation. Visualisation of the actin and microtubule cytoskeleton using microscopy identified no changes in microtubule coiling in cucurbitacin-treated platelets compared with vehicle-treated controls ( - Supplementary Fig. S4, available in the online version).

The process of platelet cytoskeleton rearrangements is dependent on proteins that bind to actin and tubulin to facilitate their polymerisation. Phosphorylation of MLCs on Ser19 enables interaction of Myosin II with actin filaments which is required for platelet shape change. In further support of an increase in actin polymerisation and alteration of cytoskeleton dynamics we observed an increase in MLC phosphorylation following treatment of platelets with cucurbitacins compared with vehicle-treated controls, similar to that observed following stimulation of platelets with TRAP-6 (-Fig. 6D).

It has been previously demonstrated that actin polymerisation agent jasplakinolide impairs platelet function via the inhibition of integrin-mediated responses. ${ }^{40}$ Jasplakinolide is not associated with dysregulation of microtubule dynamics. To ascertain whether the observed cucurbitacin-mediated effects were due to disruption of the actin cytoskeleton, platelet adhesion and spreading assays were performed in the presence of jasplakinolide ( 1 and $5 \mu \mathrm{M}$ ) (-Supplementary Fig. S5, available in the online version). Treatment with jasplakinolide caused an inhibition of platelet spreading on fibrinogen, with approximately 30\% reduction in average platelet size, which was not further inhibited by addition of cucurbitacins. This suggests that cucurbitacins act either via a similar mechanism 
A) Vehicle

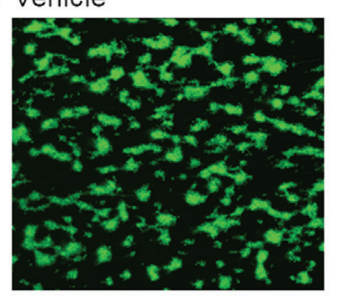

B i)

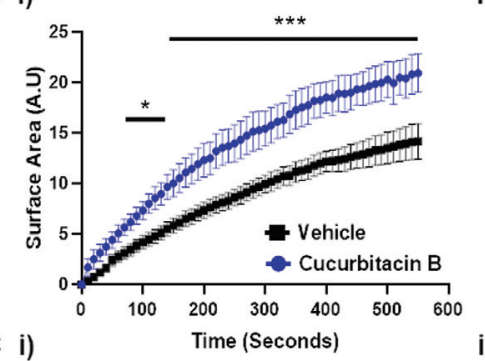

C i)

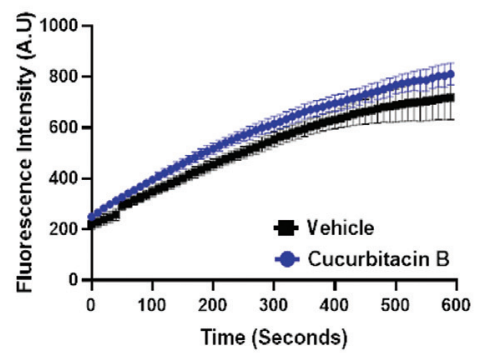

D i)

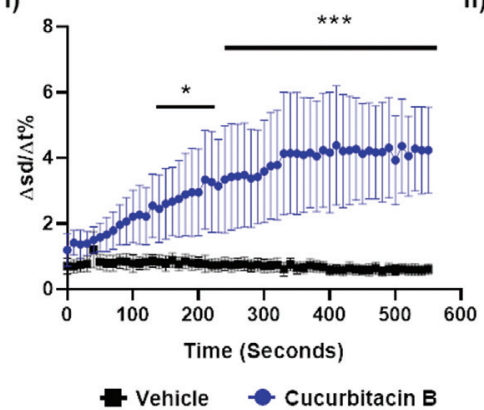

ii)

ii)


iii)

iii)
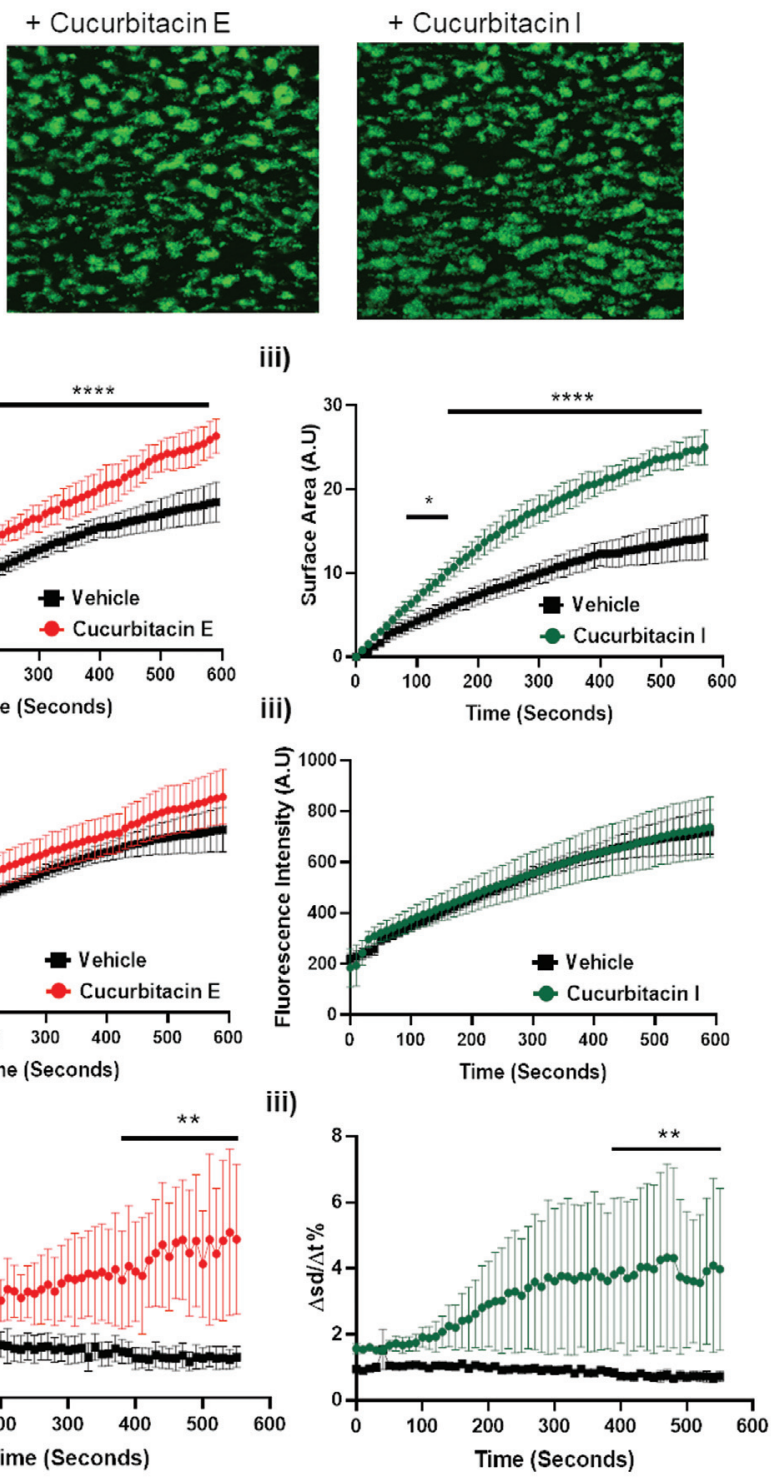

- Vehicle Cucurbitacin I

Fig. 4 Cucurbitacins inhibit stable thrombus formation on collagen in vitro. DiOC6 loaded human whole blood was pre-treated with vehicle or 10 $\mu \mathrm{M}$ cucurbitacin B, E or I for 10 minutes before perfusion through collagen-coated $(100 \mu \mathrm{g} / \mathrm{mL})$ Vena8Biochips at an arterial shear rate of 45 dynes $/ \mathrm{cm}^{2} / 1,000$ second. Thrombus formation was determined for 10 minutes. The channels were imaged on a Nikon A1-R confocal microscope to visualise thrombus formation over time, (A) representative images at 10 minutes shown. Image sequences were then analysed using ImageJ to determine (B) surface area coverage, (C) fluorescence intensity and (D) thrombus instability index (dSd/dT (\%)) compared with vehicle. (D) Representative images taken at end of each video. Results are expressed as mean \pm standard error of the mean (SEM) for $n \geq 4$. ${ }^{*}$ indicates $p<0.05$ in comparison to vehicle controls, where normalised data shown, statistics were performed prior to normalisation. Data was analysed using two-way analysis of variance (ANOVA).

to jasplakinolide, disrupting actin polymerisation and cytoskeleton dynamics or that the mechanism is masked in the presence of the actin stabilising agent.

\section{Cucurbitacins do not alter Procoagulant Platelet or Microvesicle Formation}

The actin cytoskeleton plays a key role in platelet structural integrity. Reorganisation of the cytoskeleton could have the potential to favour membrane budding and microvesicle release. ${ }^{41}$ To determine whether treatment with the cucurbitacins altered platelet procoagulant activity and the ability of platelets to form microvesicles, the effect of cucurbitacin treatment on PS exposure and platelet-derived microvesicle formation was determined in platelets in the presence and absence of the calcium ionophore A23187 (10 $\mu \mathrm{M})$. As shown in -Fig. 7, treatment with cucurbitacins alone did not induce an increase in annexin $\mathrm{V}$ binding in the absence of platelet agonist stimulation, and we observed no alteration of annexin $\mathrm{V}$ binding (\% positive cells and mean fluorescence intensity) in A23187 stimulated platelets following treatment with cucurbitacins compared with vehicle-treated controls. It has been previously demonstrated that actin polymerisation agent jasplakinolide increases platelet microvesicle formation due to its disruption of cytoskeletal dynamics. ${ }^{41}$ Interestingly, 

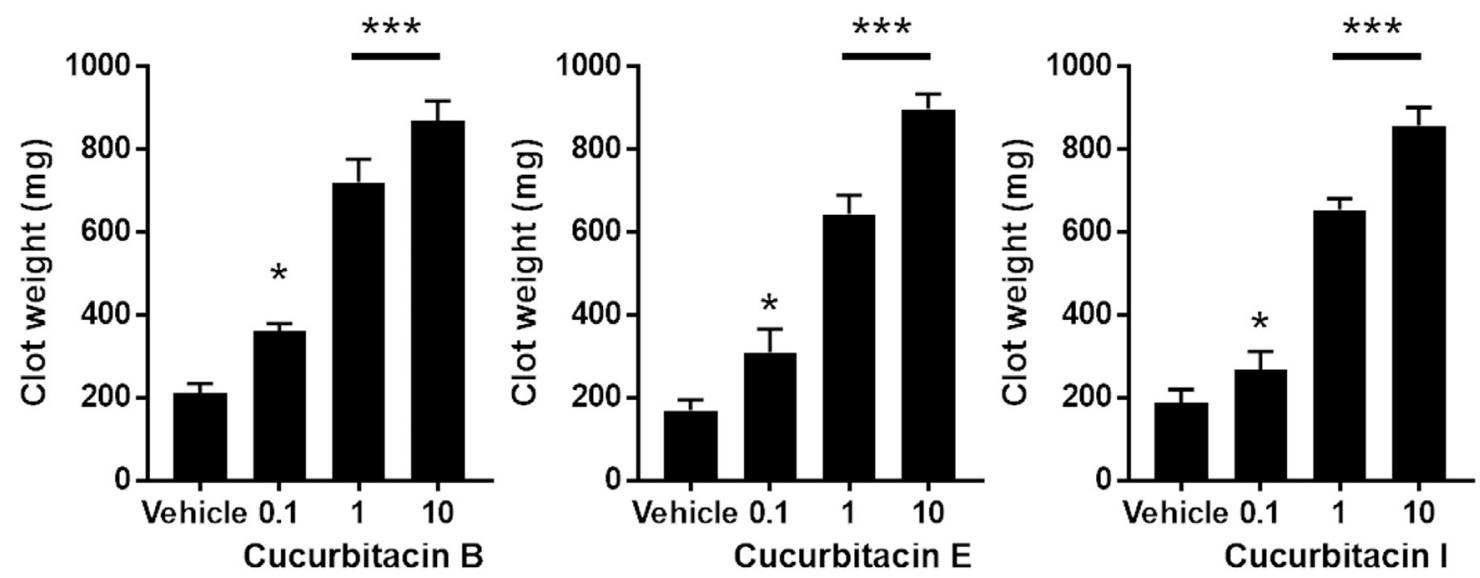

Fig. 5 Cucurbitacins inhibit clot retraction. Human washed platelets pre-treated for 10 minutes with or without increasing concentrations of cucurbitacin B, E or I $(0.1-10 \mu \mathrm{M})$ or vehicle control were added to aggregometer tubes in the presence of $2 \mathrm{mg} / \mathrm{mL} \mathrm{fibrinogen}$ and $2 \mathrm{mM}$ CaCl 2 . Clot retraction was initiated by adding $1 \mathrm{U} / \mathrm{mL}$ thrombin and left to proceed for 90 minutes at room temperature. Clot retraction was determined by weighing the clot and data expressed as percentage of vehicle-treated control. Results are mean + standard error of the mean (SEM) for $n \geq 3$, * indicates $p \leq 0.05$ in comparison to vehicle control, where normalised data shown, statistics were performed prior to normalisation.

we observed no cucurbitacin-mediated increase in microvesicle formation in unstimulated platelets, and no alteration in agonist-mediated platelet microvesicle formation following stimulation with calcium ionophore A23187. Taken together, these observations indicate that despite their ability to alter platelet cytoskeleton dynamics, cucurbitacin B, E and I unlike other actin destabilising agents, do not alter platelet procoagulant activity and microvesicle formation.

\section{Cucurbitacins Up-Regulate Cofilin Activity}

Protein kinase A (PKA) is a known mediator of Rho/ROCK signalling and a key mediator of cytoskeletal rearrangements via regulating phosphorylation of MLC. However, we show that cucurbitacins do not increase or alter PKA activity as determined by monitoring VASP S157 phosphorylation (the PKA phosphorylation site) which is unaffected by treatment with cucurbitacins (-Fig. 8A). Previously published papers have identified activation of the Rho/ROCK pathway independently of PKA following treatment with cucurbitacin I. ${ }^{42}$ ROCK kinase phosphorylates and deactivates MYPT at Thr850 which leads to increased phosphorylation of MLC on S19. ${ }^{12,43}$ However, under the same conditions that cause an increase in MLC S19 phosphorylation we observed no increase in MYPT phosphorylation compared with vehicle-treated controls ( $\mathbf{- F i g . ~ 8 B}$ ). Taken together, these data indicate cucurbitacin-induced regulation of platelet cytoskeletal changes occurs independently of PKA and RhoA/ROCK activity.

Platelet shape change requires the input of a variety of actin filament-related proteins that regulate actin cytoskeleton dynamics. Cofilin is an 'actin dynamising factor' which binds to actin filaments stabilising F actin. Due to its multiple activities, cofilin can favour both actin depolymerisation and polymerisation. To ascertain whether treatment with cucurbitacins altered cofilin activation, phosphorylation of cofilin at Ser3 was determined in unstimulated platelets following treatment with cucurbitacins B, E or I. As described by Falet et al, we observed phosphorylation of cofilin at Ser3 in vehicle-treated unstimulated platelets. We demonstrated that treatment with either cucurbitacin B, E or I caused a reduction in cofilin ser3 phosphorylation compared with vehicle-treated controls, and similar to that observed following stimulation of platelets with TRAP-6 peptide (10 uM) (-Fig. 8C). Dephosphorylation of cofilin at Ser3 has been shown to accelerate actin filament turnover, further supporting our observations that cucurbitacins disrupt actin cytoskeleton dynamics in platelets.

Cofilin is phosphorylated and inactivated by Lim kinase family LIMK-1 and 2. ${ }^{43}$ Treatment with cucurbitacins and subsequent reduced phosphorylation of cofilin indicates inhibition of LIMK-1 and/or activation of cofilin phosphatase slingshot (SSH1L). To determine whether alteration of platelet actin cytoskeleton dynamics is due to regulation of LIMK activity, phosphorylation of LIMK at Thr 508/505, a marker of activation, was determined. As shown in - Fig. 7D, no significant increase or decrease in LIMK Thr 508/505 phosphorylation was observed following treatment with cucurbitacins B, E and I compared with vehicle-treated control in resting platelets. No increase in LIMK phosphorylation is consistent with cofilin activation, but a lack of a decrease in LIMK phosphorylation indicates cucurbitacin-induced cofilin activation is not due to upstream inhibition of LIMK activity, and possibly points to an increase in SSH1L activity.

In summary, cucurbitacins B, E and I increase cofilin dephosphorylation and activation leading to dysregulation of cytoskeleton dynamics, inhibition of platelet shape change and integrin-mediated events, resulting in inhibition of stable thrombus formation.

\section{Discussion}

Compounds that target integrin function or integrin signalling pathways, preventing fibrinogen binding, platelet aggregation and thrombus formation are promising anti-platelet agents. ${ }^{44}$ However, despite their early promise as potent inhibitors of platelet aggregation, oral platelet integrin $\alpha$ IIb $\beta 3$ inhibitors 
A i)

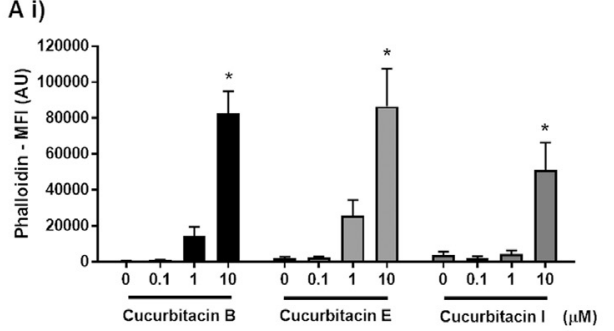

B i)

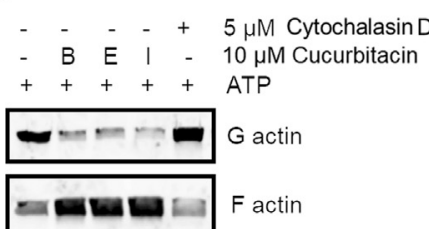

D i)

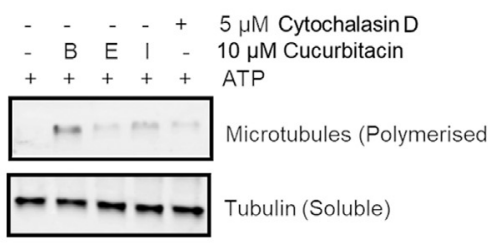

ii)
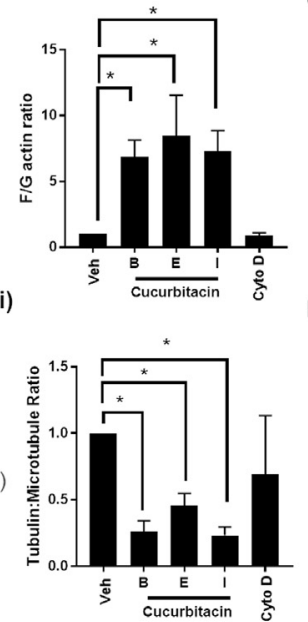

ii)
C i)

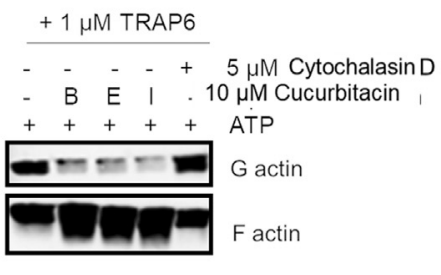

E i)

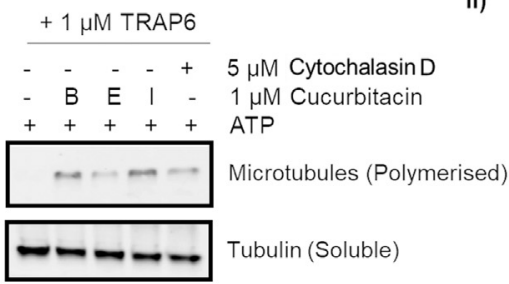

ii)
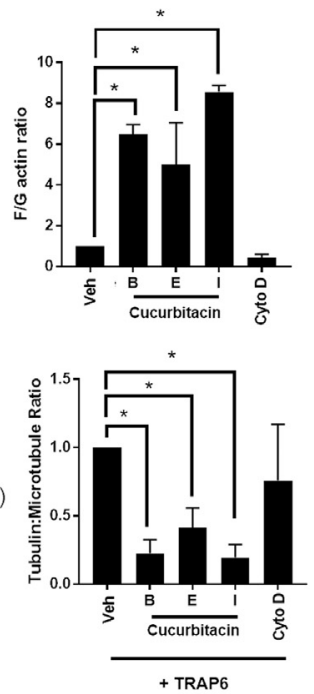

+ TRAP6
$\mathrm{F}$ i)

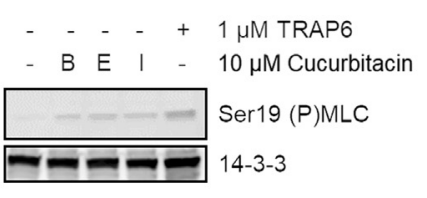

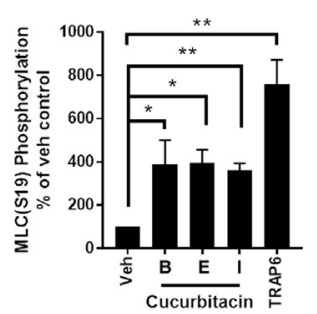

Fig. 6 Cucurbitacins alter cytoskeletal dynamics. Human washed platelets were (A) pre-treated for 10 minutes with increasing concentrations of cucurbitacin B, E or I $(0.1-10 \mu \mathrm{M})$ or vehicle before fixing, permeabilising and staining with AlexaFluor 488-conjugated phalloidin. Phalloidin staining was measured by flow cytometry, quantified data shown, (B-E) pre-treated for 10 minutes with or without cucurbitacin B, E or I (10 $\mu \mathrm{M})(\mathrm{C})$ and then stimulated with ( $\mathrm{C}$ and $\mathrm{E}$ ) thrombin receptor-activating peptide-6 (TRAP-6) $(1 \mu \mathrm{M})$ for 3 minutes and reactions stopped by lysis in Triton-X lysis buffer. The Triton insoluble pellet (polymerised $F$ actin) was then separated from the soluble fraction (monomeric $G$ actin) by centrifugation. (B and $C$ ) The $F$ and $G$ actin ratio was then calculated under each treatment condition. Cytochalasin D (an inhibitor of actin polymerisation) was included as a control. (i) Representative blot shown. (ii) F/G actin ratio in each sample were quantified and expressed as a fold change over resting control. (D and E) Under the same conditions the tubulin: microtubule ratio was then calculated for each sample, (i) Representative blot shown. (ii) tubulin:microtubule ratio in each sample were quantified and expressed as a fold change compared with resting control. (F) Human washed platelets were pre-treated for 10 minutes with or without cucurbitacin B, E or I $(1 \mu \mathrm{M})$ and reactions stopped by lysis in sodium dodecyl sulfate (SDS) Laemmli sample buffer and myosin light chain phosphorylation at Ser19 determined using a phospho-specific antibody (that recognises the phosphorylated myosin light chain). TRAP-6 (1 $\mu \mathrm{M})$-stimulated platelets were included as a positive control for activation, 14-3-3 was included as a loading control. (i) Representative blot shown. (ii) Quantified data expressed as a percentage of untreated control. Results represent mean + standard error of the mean (SEM) for $n \geq 3$. * Indicates $p<0.05$ in comparison with vehicle control, where normalised data are shown, statistics were performed prior to normalisation.

show no benefit and/or increased mortality rate in clinical trials. $^{45,46}$ New approaches that indirectly target integrin function could therefore offer a safer, more efficacious approach to integrin $\alpha \operatorname{Ilb} \beta 3$ targeted anti-platelet therapy. Novel therapies targeting regulation of platelet shape change and cytoskeleton rearrangements are also of increasing interest. ${ }^{21}$

Cucurbitacins, tetracyclic triterpernoid compounds, have been shown to elicit a range of beneficial properties including anti-cancer, anti-diabetic, anti-inflammatory, anti-oxidant and anti-atherosclerotic activities through a variety of mechanisms including regulation of cofilin, dysregulation of actin cytoskeleton dynamics ${ }^{11,12,16}$ and disruption of integrin function. ${ }^{17,18}$
We observed anti-platelet activity of cucurbitacins B, E and I which results in a significant attenuation in the ability of platelets to form stable thrombi. Most notably, while initial platelet adhesion to collagen (and fibrinogen) under flow appeared to be unaffected by treatment with cucurbitacins, the morphology of the thrombi was altered, with frequent embolisation observed and clot retraction perturbed. Analysis of thrombus stability using the thrombus instability index $\Delta \mathrm{Sd} / \Delta \mathrm{T}(\%)^{31}$ identified increased $\Delta \mathrm{Sd} / \Delta \mathrm{T}$ (\%) values following cucurbitacin treatment, indicating a reduction in stable thrombi formation. These observations are likely resultant from the actions of cucurbitacins on the 
A)

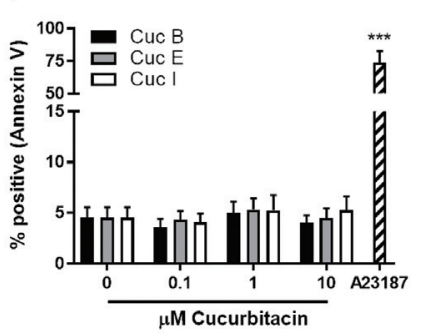

C i)

Unstimulated

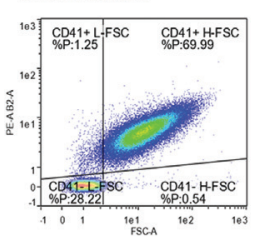

B i)

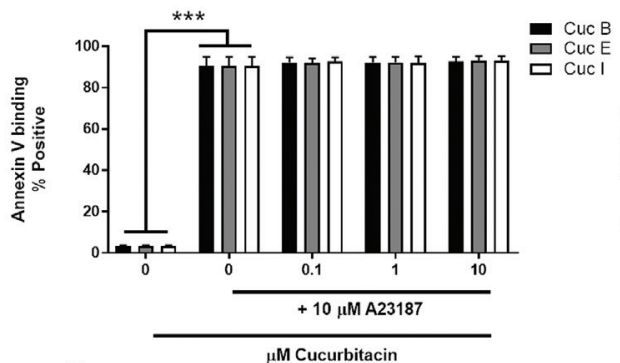

ii)

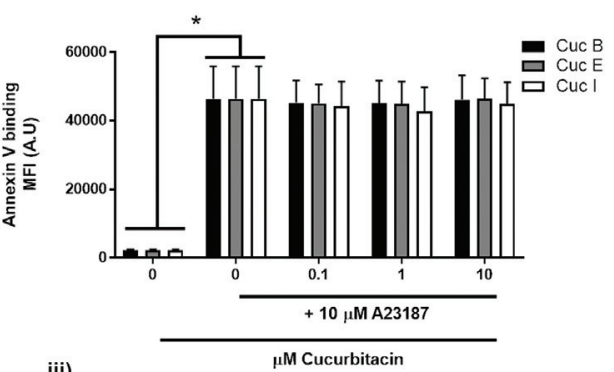

iii)
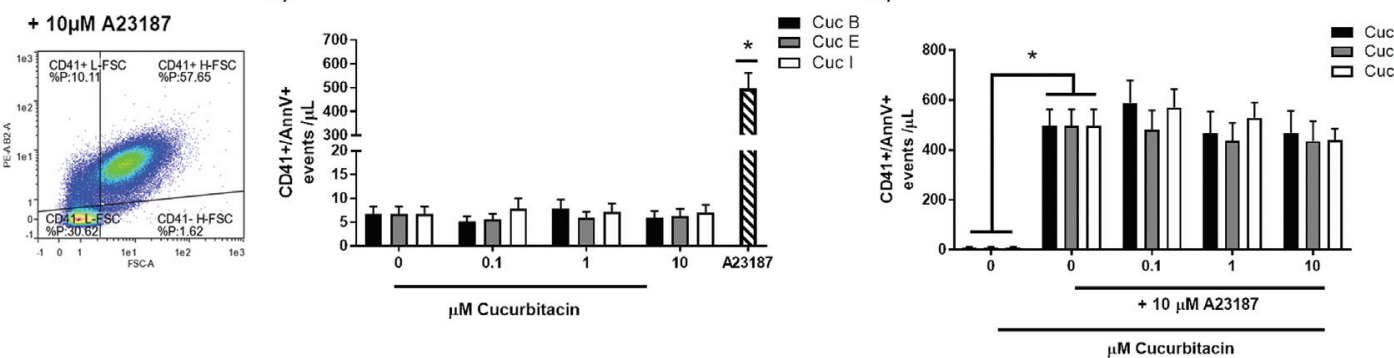

Fig. 7 Cucurbitacins do not initiate procoagulant platelet or microvesicle formation. Human washed platelets pre-treated for 10 minutes with or without cucurbitacin B, E or I ( $1 \mu \mathrm{M})$ for 10 minutes prior to stimulation with $10 \mu \mathrm{M}$ A23817 for 10 minutes at room temperature and (A and B) phosphatidylserine exposure determined by measuring (A) and (B) (i) quantified data for annexin $\mathrm{V} \%$ positive platelets and (B) (ii) quantified data for median fluorescence intensity using fluorescein isothiocyanate (FITC)-conjugated annexin V shown. (C) Microvesicle formation following treatment with or without cucurbitacins in the (i) absence and (ii) presence of calcium ionophore $(10 \mu \mathrm{M} \mathrm{A23817)}$ was determined by counting the number of CD41 + /annexin $\mathrm{V}+$ events per $\mu \mathrm{L}$ using flow cytometry and staining with an phycoerythrin (PE)-conjugated anti-human CD41a antibody and FITC-conjugated annexin V. (i) Representative histogram plots demonstrating the gating strategy based on forward scatter (FSC) and CD41+ events, (ii) and (iii) quantified data shown. When samples were treated with cucurbitacins alone in the absence of ionophore stimulation ( $\mathrm{A}$ and C (i)), $10 \mu \mathrm{M} \mathrm{A23817}$ was included as a positive control. Data was collected using a MACsQuant Analyser 16 flow cytometer and analysed using FlowLogic software. Results are mean + standard error of the mean (SEM) for $n \geq 3$, *indicates $p<0.05,{ }^{* *}$ indicates $p<0.01,{ }^{* * *} p<0.001$ in comparison with vehicle control.

cytoskeleton, including alteration of actin dynamics and microtubule organisation.

Following treatment with cucurbitacins we observed an increase in actin polymerisation, $F$ actin/ $G$ actin ratio, a decrease in tubulin:microtubule ratio and increase in MLC phosphorylation. This occurred independently of platelet agonist activation, indicating that cytoskeletal dynamics and actin and microtubule turnover are arrested at the stage of polymerisation. Consistent with this, platelet adhesion and spreading on a range of surfaces, a process dependent on cytoskeletal remodelling and polymerisation, was disrupted by cucurbitacins. Furthermore, clot retraction, which is driven by the cytoskeletal tensile strength and dynamics, was also inhibited by cucurbitacins B, E and I. Interestingly, we did observe some differences in the concentrations of cucurbitacins required to cause inhibition of functional responses. Treatment with higher concentrations of cucurbitacins was needed to inhibit platelet aggregation compared with platelet spreading and cytoskeleton rearrangements. We hypothesise that this is due to the differences in the nature of the assays and their reliance on cytoskeleton rearrangements.

The mode of action of cucurbitacins observed in this study is different to that observed following treatment with latrunculin A or cytochalasin D, inhibitors of actin polymerisation. Instead, our observations align with those made previously using jasplakinolide, a stabiliser of actin filaments. ${ }^{40,47}$ This is supported by experiments that show cucurbitacins are unable to further inhibit jasplakinolide-induced inhibition of platelet spreading (-Supplementary Fig. S3, available in the online version).
Jasplakinolide induces actin polymerisation by promoting actin nucleation and preventing depolymerisation of actin stress fibres. Bennett et al and Bury et al describe how treatment with jasplakinolide leads to impairment of platelet function, specifically as a result of impaired $\alpha_{\mathrm{II}} \beta_{3}$ activation, reduced aggregation, altered spreading on fibrinogen and clot retraction, observations that are similar to what we observed following treatment with cucurbitacins. Like our observations with cucurbitacins B, E and I, Bury et al also identify that actin polymerisation (following treatment with jasplakinolide) does not alter Pselectin exposure. ${ }^{40}$ However, further investigation did identify cucurbitacin mediated inhibition of PF4 release, under conditions where cucurbitacins inhibit platelet aggregation, indicating cucurbitacin-mediated disruption of the actin dynamics reduces the release of soluble $\alpha$-granule contents. In contrast to our findings with the cucurbitacins, Bennett et al demonstrate jasplakinolide does not alter dense granule secretion, while we observed decreased CD63 exposure following treatment with cucurbitacins. This could be reflective of differences in the mechanism by which the two groups of compounds elicit actin polymerisation, although it should also be noted that Bennett et al, measured serotonin release to $2 \mathrm{U} / \mathrm{mL}$ thrombin which is a very high concentration of the strong activatory agonist, and therefore is likely difficult to inhibit.

We also observed no alteration of platelet-derived microvesicle formation following treatment with cucurbitacins. This is a particularly promising observation as other actin disrupting agents are associated with increased microvesicle formation. ${ }^{41}$ Platelet-derived procoagulant microvesicles 

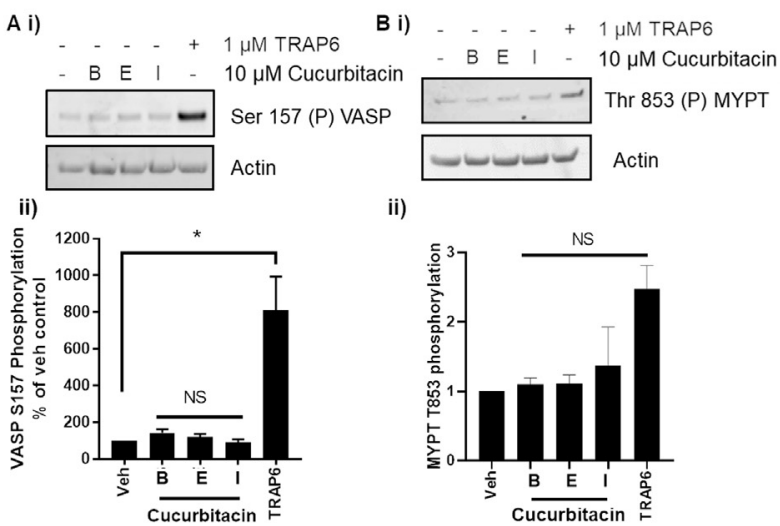

ii)

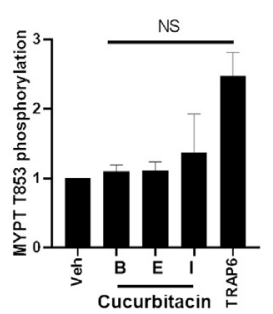

C i)



ii)

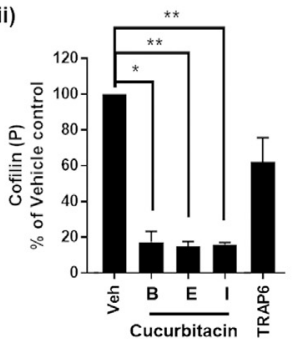

D i)
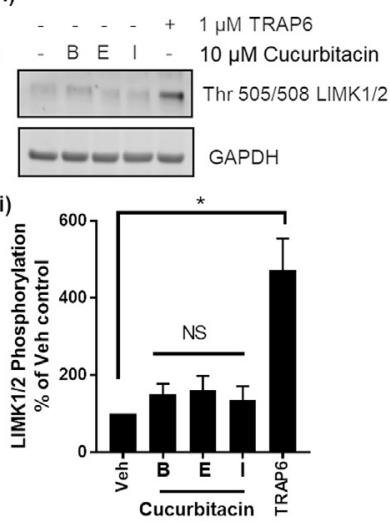

Fig. 8 Cucurbitacins regulate cofilin activity. Human washed platelets were pre-treated for 10 minutes with or without cucurbitacin $B, E$ or I ( $10 \mu \mathrm{M})$ or for 3 minutes with thrombin receptor-activating peptide-6 (TRAP-6) ( $1 \mu \mathrm{M})$ (positive control) and reactions stopped by lysis in sodium dodecyl sulfate (SDS) Laemmli sample buffer and (A) protein kinase $A$ (PKA) activity was determined by blotting these samples for vasodilator-stimulated phosphoprotein (VASP) S157 phosphorylation, (B) myosin light chain phosphatase activity determined by blotting samples for MYPT T853 phosphorylation, (C) cofilin activity was determined by blotting these samples for Ser3 phosphorylation and (D) LIMK1/2 activity was determined by blotting these samples for LIMK1/2 Thr 505/508 phosphorylation using phospho-specific antibodies. Actin, cofilin and glyceraldehyde 3phosphate dehydrogenase (GAPDH) were used to confirm equal loading respectively. (i) Representative blots and (ii) quantified data shown. Levels of total phosphorylation were quantified and expressed as a percentage of the vehicle control. Results are mean + standard error of the mean (SEM) for $n \geq 3$, *indicates $p<0.05$, **indicates $p<0.01$ in comparison with vehicle control, where normalised data shown, statistics were performed prior to normalisation.

have been shown to enhance coagulation and thrombosis, which could potentially outweigh any anti-platelet activity of other actin disrupting agents. Specific therapeutic targeting of the platelet cytoskeleton and enhancing thrombus destabilisation opens new avenues for the development of novel anti-platelet therapies. The lack of effect on microvesicle formation and platelet procoagulant activity indicates that the cucurbitacins are a suitable scaffold for developing novel anti-platelet agents.

Several publications in other cell types have identified that cucurbitacins cause an increase and stabilisation of polymer-

ised actin, resulting in a condensed actin network. ${ }^{11,12,16,42}$ Possible mechanisms of action include regulation of actin binding or actin remodelling proteins, or via direct interaction with and stabilisation of the actin cytoskeleton, however, the precise mechanism is still not fully understood.

Previous publications have identified cucurbitacin mediated activation of the Rho/ROCK pathways. ${ }^{12,42}$ This pathway in platelets regulates cytoskeleton dynamics via deactivation of MYPT by phosphorylation (at Thr850), increased phosphorylation of the MLC at S19 and increased actin polymerisation. ${ }^{48}$ However, we observed no increase in MYPT Thr850 phosphorylation following treatment with the cucurbitacins indicating an alternative mechanism of action.

Cofilin activity and subsequent actin depolymerisation has been proposed to be altered following treatment with cucurbitacins in other cell types both via direct and indirect mechanisms. ${ }^{11,12}$ Cofilin acts as an 'actin dynamising factor' which binds to actin filaments stabilising Factin, where depending on the cellular content of actin filaments, cofilin can favour actin depolymerisation or polymerisation. We identified reduced phosphorylation at Ser3 and activation of cofilin following treatment with cucurbitacins in platelets. Both Falet et $\mathrm{al}^{49}$ and Pandey et $\mathrm{al}^{43}$ identified that cofilin dephosphorylation is accompanied by an increase in F-actin content and increased association of cofilin with F-actin. Taken together, these findings indicate that cucurbitacins alter actin polymerisation and cytoskeleton dynamics via regulation of cofilin dephosphorylation and activity.

Cofilin is phosphorylated and inactivated by LIMK- 1 and $2^{43}$ downstream of the RhoA/PKA/VASP signalling pathway and activated by dephosphorylation via cofilin phosphatase slingshot (SSH1L). Cucurbitacins have been shown to induce stimulation of the small GTPases observed in other cell types. ${ }^{40,50}$ However, in support of lack of activation of the RhoA/PKA/VASP-LIMK-1 pathway in platelets, we observed no alteration in VASP S157 phosphorylation, or phosphorylation of LIMK at Thr 508/505, following treatment with cucurbitacins B, E or I in resting platelets, indicating no activation of PKA or LIMK activity. This is further supported by our observations that treatment with cucurbitacins B, E or I does not alter platelet cytosolic calcium mobilisation or granule secretion, processes known to be negatively regulated by increases in PKA activity.

Similar to our observations, a study investigating the anticancer properties of cucurbitacins using HeLa cells identified that cucurbitacin I induced the formation of actin and myosin II coaggregates that led to an alteration in cytoskeletal dynamics. ${ }^{40}$ Interestingly, this study identified reduced cofilin phosphorylation which the authors attributed to an inhibition of LIMK via direct binding of cucurbitacin I to the kinase. In contrast, our observations of a lack of increase in both PKA and LIMK activity is consistent with cofilin activation, but indicates that cucurbitacin-mediated cofilin activation is likely associated with either an increase in SSH1L phosphatase activity which leads to cofilin dephosphorylation, or activation of an alternative regulatory pathway that results in a negative regulation of cofilin 


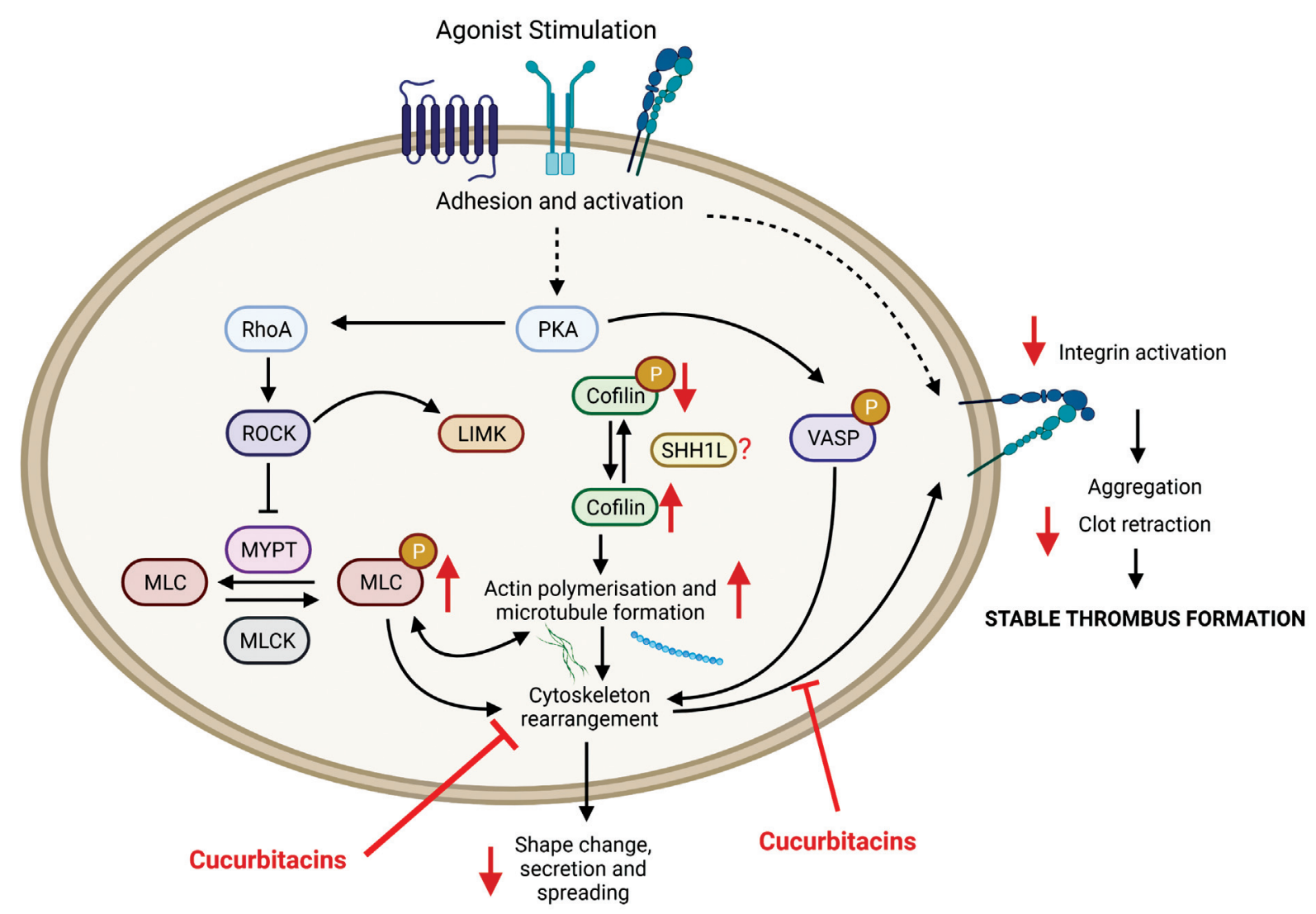

Fig. 9 Cucurbitacins perturb actin cytoskeleton rearrangements and integrin function in platelets. Cucurbitacins inhibit stable thrombus formation via disruption of platelet cytoskeleton dynamics. Cucurbitacins increase the platelet $\mathrm{F} / \mathrm{G}$ actin ratio, actin polymerisation and microtubule formation, alongside up-regulation of cofilin activity and myosin light chain phosphorylation via a mechanism which appears to be independent of regulation of protein kinase A (PKA), ROCK or LIMK. Created with BioRender.com

phosphorylation and subsequent activation (summarised in -Fig. 9).

Our research identifies novel, anti-platelet and antithrombotic actions of cucurbitacins that appear to be linked to cofilin-mediated dysregulation of cytoskeletal dynamics, and subsequent inhibition of integrin activity (-Fig. 9). Therapeutic targeting of the platelet cytoskeleton and thrombus destabilisation is an attractive novel approach for anti-platelet therapy.

At this stage it should be noted that these compounds are associated with toxicity issues at therapeutic doses, and therefore in their current form have limited pharmacological use. This is not surprising nor is it a major cause of concern, noting that natural products have provided chemical leads for the development of many drugs for a range of indications. ${ }^{51}$ Due to the molecular complexity of the cucurbitacins scaffold, intelligent compound design and pharmacophore modelling will allow us to simplify the scaffold into more 'drug-like' motifs, ${ }^{52}$ incorporating covalent warheads, ${ }^{53}$ shape complementarity ${ }^{54}$ and functional group mapping 55 to develop novel more 'drug-like' compounds capable of eliciting a similar biological response.

Cucurbitacin-derived compounds ${ }^{1}$ therefore represent an exciting scaffold for a novel anti-platelet targeting strategy that can control and inhibit platelet function by preferen- tially targeting the formation of stable platelet aggregates and thrombi.

\section{What is known about this topic?}

- Natural compound libraries produced from plant products have identified a variety of promising compounds for further development as therapeutic agents.

- Cucurbitacins are dietary compounds that have been shown to elicit a range of anti-tumour, anti-inflammatory and anti-atherosclerotic activities.

- The effects of cucurbitacins on platelet function and thrombus formation are unknown.

\section{What does this paper add?}

- This research identifies novel, anti-platelet and antithrombotic actions of cucurbitacins.

- Cucurbitacins disrupt platelet cytoskeletal dynamics, increasing actin polymerisation, microtubule formation, myosin light chain activation and cofilin activity.

- Cucurbitacins potently inhibit integrin- and cytoskeleton-mediated events, including adhesion, spreading, clot retraction and stable thrombus formation. 


\section{Author Contributions}

Contribution: N.K., S.N., T.S., B.K., A.P.B., J.L.M., S.T., performed experiments and analysed results. M.G.M., S.J. and J.M.G. designed the research and wrote the paper. A.J.U. designed the research, performed experiments, analysed results and wrote the paper.

\section{Funding}

This work was supported by a British Heart Foundation Project Grant PG/2019/34798 (to A.J.U.) and British Heart Foundation programme grants RG/15/2/31224 and RG/ 20/7/34866 (to J.M.G.), and research funds from the Centre for Bioscience at Manchester Metropolitan University and a Manchester Metropolitan University Internal Research Accelerator Grant: 343846 (to A.J.U.).

\section{Conflict of Interest}

None declared.

\section{Acknowledgements}

The authors would like to thank Dr. Nicholas Pugh, Anglia Ruskin University, for assistance with analysis of the thrombus formation stability index and preparation of this manuscript, Dr. Stephen White, Manchester Metropolitan University for provision of reagents and Andy Bashford and Simon Lydford, Molecular Devices (Winnersh, U.K.) for access to and assistance with the Pico Imaging equipment.

\section{References}

1 Kaushik U, Aeri V, Mir SR. Cucurbitacins - an insight into medicinal leads from nature. Pharmacogn Rev 2015;9(17):12-18

2 Stainer AR, Sasikumar P, Bye AP, et al. The metabolites of the dietary flavonoid quercetin possess potent antithrombotic activity, and interact with aspirin to enhance antiplatelet effects. TH Open 2019;3(03):e244-e258

3 Vaiyapuri S, Ali MS, Moraes LA, et al. Tangeretin regulates platelet function through inhibition of phosphoinositide 3-kinase and cyclic nucleotide signaling. Arterioscler Thromb Vasc Biol 2013; 33(12):2740-2749

4 Vaiyapuri S, Roweth H, Ali MS, et al. Pharmacological actions of nobiletin in the modulation of platelet function. $\mathrm{Br} \mathrm{J}$ Pharmacol 2015;172(16):4133-4145

5 Ed Nignpense B, Chinkwo KA, Blanchard CL, Santhakumar AB. Polyphenols: modulators of platelet function and platelet microparticle generation? Int J Mol Sci 2019;21(01):E146

6 Kaneider NC, Mosheimer B, Reinisch N, Patsch JR, Wiedermann CJ. Inhibition of thrombin-induced signaling by resveratrol and quercetin: effects on adenosine nucleotide metabolism in endothelial cells and platelet-neutrophil interactions. Thromb Res 2004;114(03):185-194

7 Chen X, Bao J, Guo J, et al. Biological activities and potential molecular targets of cucurbitacins: a focus on cancer. Anticancer Drugs 2012;23(08):777-787

8 Alghasham AA. Cucurbitacins - a promising target for cancer therapy. Int J Health Sci (Qassim) 2013;7(01):77-89

9 Blaskovich MA, Sun J, Cantor A, Turkson J, Jove R, Sebti SM. Discovery of JSI-124 (cucurbitacin I), a selective Janus kinase/signal transducer and activator of transcription 3 signaling pathway inhibitor with potent antitumor activity against human and murine cancer cells in mice. Cancer Res 2003;63(06): $1270-1279$
10 Sun J, Blaskovich MA, Jove R, Livingston SK, Coppola D, Sebti SM. Cucurbitacin Q: a selective STAT3 activation inhibitor with potent antitumor activity. Oncogene 2005;24(20): 3236-3245

11 Nakashima S, Matsuda H, Kurume A, et al. Cucurbitacin E as a new inhibitor of cofilin phosphorylation in human leukemia U937 cells. Bioorg Med Chem Lett 2010;20(09):2994-2997

12 Zhang YT, Xu LH, Lu Q et al. VASP activation via the Go13/RhoA/PKA pathway mediates cucurbitacin-B-induced actin aggregation and cofilin-actin rod formation. PLoS One 2014;9(04): e93547

13 Kong Y, Chen J, Zhou Z, Xia H, Qiu MH, Chen C. Cucurbitacin E induces cell cycle $\mathrm{G} 2 / \mathrm{M}$ phase arrest and apoptosis in triple negative breast cancer. PLoS One 2014;9(07):e103760

14 Jayaprakasam B, Seeram NP, Nair MG. Anticancer and antiinflammatory activities of cucurbitacins from Cucurbita andreana. Cancer Lett 2003;189(01):11-16

15 McFarland BC, Gray GK, Nozell SE, Hong SW, Benveniste EN. Activation of the NF-KB pathway by the STAT3 inhibitor JSI-124 in human glioblastoma cells. Mol Cancer Res 2013;11(05): 494-505

16 Wang X, Tanaka M, Peixoto HS, Wink M. Cucurbitacins: elucidation of their interactions with the cytoskeleton. PeerJ 2017;5: e3357

17 Touihri-Barakati I, Kallech-Ziri O, Ayadi W, et al. Cucurbitacin B purified from Ecballium elaterium (L.) A. Rich from Tunisia inhibits $\alpha 5 \beta 1$ integrin-mediated adhesion, migration, proliferation of human glioblastoma cell line and angiogenesis. Eur J Pharmacol 2017;797:153-161

18 Gupta P, Srivastava SK. Inhibition of Integrin-HER2 signaling by Cucurbitacin B leads to in vitro and in vivo breast tumor growth suppression. Oncotarget 2014;5(07):1812-1828

19 Gong H, Shen B, Flevaris P, et al. G protein subunit Galpha13 binds to integrin alphallbbeta3 and mediates integrin "outside-in" signaling. Science 2010;327(5963):340-343

20 Durrant TN, van den Bosch MT, Hers I. Integrin $\alpha_{\mathrm{IIb}} \beta_{3}$ outside-in signaling. Blood 2017;130(14):1607-1619

21 Shin EK, Park H, Noh JY, Lim KM, Chung JH. Platelet shape changes and cytoskeleton dynamics as novel therapeutic targets for antithrombotic drugs. Biomol Ther (Seoul) 2017;25(03):223-230

22 Bye AP, Unsworth AJ, Gibbins JM. Platelet signaling: a complex interplay between inhibitory and activatory networks. J Thromb Haemost 2016;14(05):918-930

$23 \mathrm{Ni} \mathrm{H}$, Freedman J. Platelets in hemostasis and thrombosis: role of integrins and their ligands. Transfus Apheresis Sci 2003;28(03): 257-264

24 Moraes LA, Spyridon M, Kaiser WJ, et al. Non-genomic effects of PPARgamma ligands: inhibition of GPVI-stimulated platelet activation. J Thromb Haemost 2010;8(03):577-587

25 Vaiyapuri S, Jones CI, Sasikumar P, et al. Gap junctions and connexin hemichannels underpin hemostasis and thrombosis. Circulation 2012;125(20):2479-2491

26 Wei H, Davies JE, Harper MT. 2-Aminoethoxydiphenylborate (2APB) inhibits release of phosphatidylserine-exposing extracellular vesicles from platelets. Cell Death Discov 2020;6:10

27 Grynkiewicz G, Poenie M, Tsien RY. A new generation of Ca2+ indicators with greatly improved fluorescence properties. J Biol Chem 1985;260(06):3440-3450

28 Poenie M. Alteration of intracellular Fura-2 fluorescence by viscosity: a simple correction. Cell Calcium 1990;11(2-3):85-91

29 Bye AP, Unsworth AJ, Vaiyapuri S, Stainer AR, Fry MJ, Gibbins JM. Ibrutinib inhibits platelet integrin $\alpha$ IIb $\beta 3$ outside-in signaling and thrombus stability but not adhesion to collagen. Arterioscler Thromb Vasc Biol 2015;35(11):2326-2335

30 Kanaji T, Kanaji S, Montgomery RR, Patel SB, Newman PJ. Platelet hyperreactivity explains the bleeding abnormality and macrothrombocytopenia in a murine model of sitosterolemia. Blood 2013;122(15):2732-2742 
31 Pugh N, Maddox BD, Bihan D, Taylor KA, Mahaut-Smith MP, Farndale RW. Differential integrin activity mediated by platelet collagen receptor engagement under flow conditions. Thromb Haemost 2017;117(08):1588-1600

32 Unsworth AJ, Bye AP, Sage T, et al. Antiplatelet properties of Pim kinase inhibition are mediated through disruption of thromboxane A2 receptor signaling. Haematologica 2021;106(07): 1968-1978

33 Bye AP, Unsworth AJ, Desborough MJ, et al. Severe platelet dysfunction in NHL patients receiving ibrutinib is absent in patients receiving acalabrutinib. Blood Adv 2017;1(26): 2610-2623

34 Pugh N, Bihan D, Perry DJ, Farndale RW. Dynamic analysis of platelet deposition to resolve platelet adhesion receptor activity in whole blood at arterial shear rate. Platelets 2015;26(03): 216-219

35 Khan AO, Slater A, Maclachlan A, et al. Post-translational polymodification of $\beta 1$-tubulin regulates motor protein localisation in platelet production and function. Haematologica 2022;107(01): 243-259

36 Wersäll A, Williams CM, Brown E, Iannitti T, Williams N, Poole AW. Mouse platelet Ral GTPases control P-selectin surface expression, regulating platelet-leukocyte interaction. Arterioscler Thromb Vasc Biol 2018;38(04):787-800

37 Flaumenhaft R, Dilks JR, Rozenvayn N, Monahan-Earley RA, Feng $D$, Dvorak AM. The actin cytoskeleton differentially regulates platelet alpha-granule and dense-granule secretion. Blood 2005;105(10):3879-3887

38 Woronowicz K, Dilks JR, Rozenvayn N, et al. The platelet actin cytoskeleton associates with SNAREs and participates in alphagranule secretion. Biochemistry 2010;49(21):4533-4542

39 Duncan MD, Duncan KL. Cucurbitacin E targets proliferating endothelia. J Surg Res 1997;69(01):55-60

40 Bury L, Falcinelli E, Chiasserini D, Springer TA, Italiano JE Jr, Gresele P. Cytoskeletal perturbation leads to platelet dysfunction and thrombocytopenia in variant forms of Glanzmann thrombasthenia. Haematologica 2016;101(01):46-56

41 Cauwenberghs S, Feijge MA, Harper AG, Sage SO, Curvers J, Heemskerk JW. Shedding of procoagulant microparticles from unstimulated platelets by integrin-mediated destabilization of actin cytoskeleton. FEBS Lett 2006;580(22):5313-5320
42 Sari-Hassoun M, Clement MJ, Hamdi I, et al. Cucurbitacin I elicits the formation of actin/phospho-myosin II co-aggregates by stimulation of the RhoA/ROCK pathway and inhibition of LIM-kinase. Biochem Pharmacol 2016;102:45-63

43 Pandey D, Goyal P, Bamburg JR, Siess W. Regulation of LIM-kinase 1 and cofilin in thrombin-stimulated platelets. Blood 2006;107 (02):575-583

44 Millard M, Odde S, Neamati N. Integrin targeted therapeutics. Theranostics 2011;1:154-188

45 Chew DP, Bhatt DL, Topol EJ. Oral glycoprotein IIb/IIIa inhibitors: why don't they work? Am J Cardiovasc Drugs 2001;1(06): 421-428

46 Cox D. Oral GPIIb/IIIa antagonists: what went wrong? Curr Pharm Des 2004;10(14):1587-1596

47 Bennett JS, Zigmond S, Vilaire G, Cunningham ME, Bednar B. The platelet cytoskeleton regulates the affinity of the integrin alpha (IIb)beta(3) for fibrinogen. J Biol Chem 1999;274(36): 25301-25307

48 Aburima A, Walladbegi K, Wake JD, Naseem KM. cGMP signaling inhibits platelet shape change through regulation of the RhoARho Kinase-MLC phosphatase signaling pathway. J Thromb Haemost 2017;15(08):1668-1678

49 Falet H, Chang G, Brohard-Bohn B, Rendu F, Hartwig JH. Integrin alpha(IIb)beta3 signals lead cofilin to accelerate platelet actin dynamics. Am J Physiol Cell Physiol 2005;289 (04):C819-C825

50 Zhang M, Bian ZG, Zhang Y, et al. Cucurbitacin B inhibits proliferation and induces apoptosis via STAT3 pathway inhibition in A549 lung cancer cells. Mol Med Rep 2014;10(06):2905-2911

51 Beutler JA. Natural products as a foundation for drug discovery. Curr Protocols Pharmacol 2019;86(01):e67

52 Guo Z. The modification of natural products for medical use. Acta Pharm Sin B 2017;7(02):119-136

53 Gehringer M, Laufer SA. Emerging and re-emerging warheads for targeted covalent inhibitors: applications in medicinal chemistry and chemical biology. J Med Chem 2019;62(12):5673-5724

54 Kumar A, Zhang KYJ. Advances in the development of shape similarity methods and their application in drug discovery. Front Chem 2018;6:315

55 Guvench O. Computational functional group mapping for drug discovery. Drug Discov Today 2016;21(12):1928-1931 